Faisons mieux les choses : représentation

publique de la science sur la COVID-19

Octobre 2020

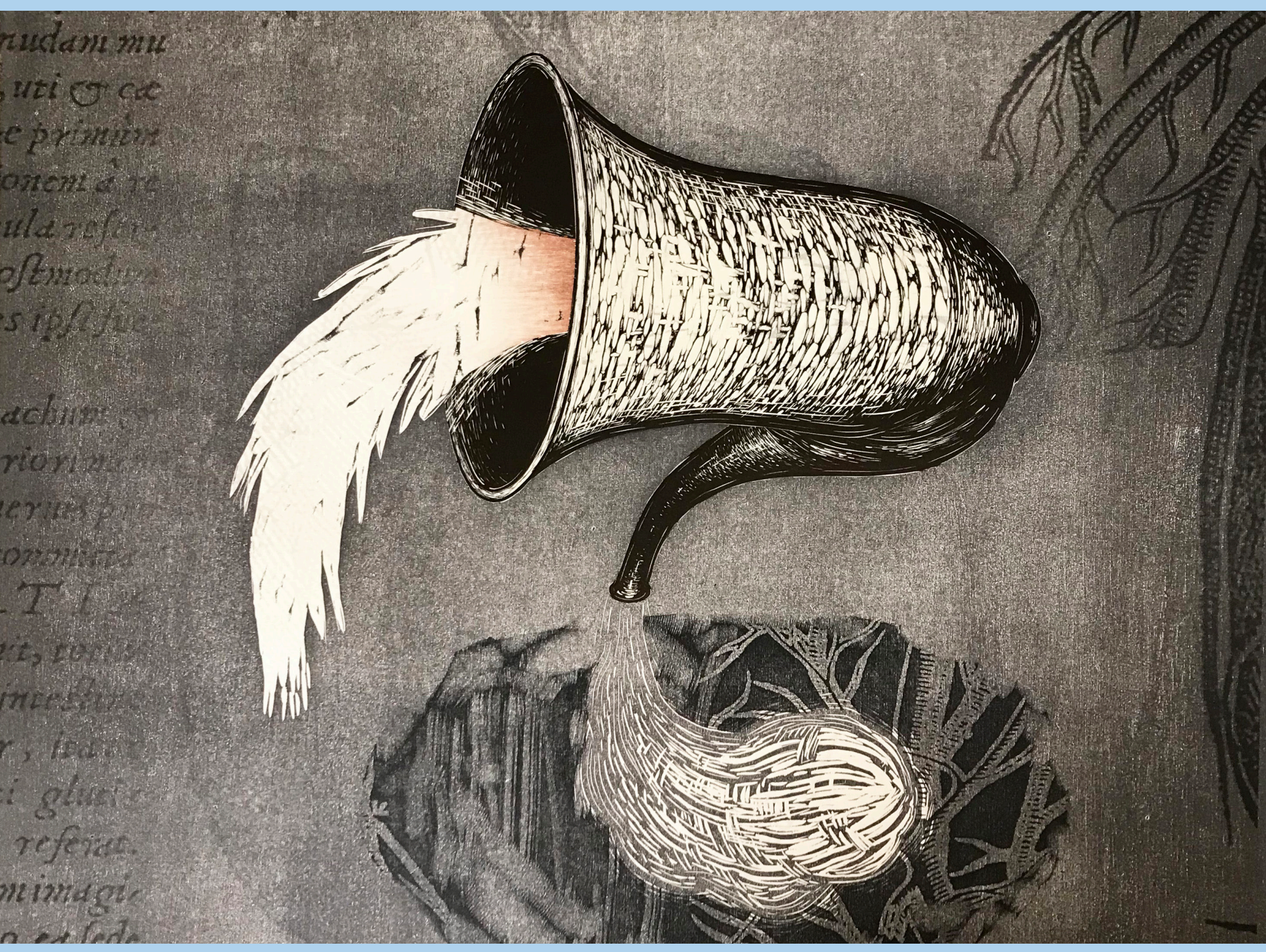




\section{Faisons mieux les choses: représentation \\ publique de la science sur la COVID-19}

Un note de breffage de la SRC

\section{Auteurs}

Tania Bubela, MSRC

Timothy Caulfield, MSRC (Président)

Jonathan Kimmelman

Vardit Ravitsky
Simon Fraser University

University of Alberta

Université McGill

Université de Montréal

Responsable de la surveillance du processus d'examen par les pairs

Tom Marrie, MSRC Dalhousie University

\section{Pairs examinateurs de la note de breffage}

Marie-Josée Hébert

Bev Holmes

Andreas Laupacis

Lynora Saxinger
Université de Montréal

Michael Smith Foundation for Health Research

St. Michael's Hospital

University of Alberta

Forme suggérée pour les citations de cette note de breffage :

Caulfield, T., Bubela, T., Kimmelman, J., Ravitsky, V. Faisons mieux les choses : représentation publique de la science sur la COVID-19. Société royale du Canada. 2020

\section{Image de couverture}

Sean Caulfield, MSRC, Think Accuracy, 58 × 58cm (2020)

Relief et jet d'encre sur papier de chiffon

Cette illustration est tirée d'Infodemic, un projet de livre d'artiste jumelant des textes et des images créés dans le cadre d'une collaboration interdisciplinaire nouée entre Sean Caulfield, professeur au Département des arts et du design de l'University of Alberta ( $U$ of $A)$, Timothy Caulfield, titulaire d'une Chaire de recherche du Canada et directeur du Health Law Institute de l'U of A et Sue Colberg, professeure agrégée au Département des arts et du design de I'U of A. Le projet examine les répercussions des fausses informations qui circulent sur les questions de santé dans le contexte de la pandémie de la COVID-19. Les illustrations ont initialement été publiées sur les médias sociaux dans l'espoir que les gens s'arrêtent et réfléchissent avant de relayer de fausses informations sur la COVID-19. Par exemple, avec cette gravure, l'artiste a voulu créer, par l'intermédiaire d'un langage abstrait, une image qui fait référence à un corps en dépeignant une forme de récipient qui pourrait évoquer une personne qui tousse ou qui crie. En même temps, la forme évoque un mégaphone qui hurle bruyamment. De façon plus générale, le projet Infodemic se veut une exploration créative du climat d'incertitude et d'anxiété que la crise de la COVID-19 a engendré.

\section{Reconnaissance territorial}

Le siège social de la Société royale du Canada est situé à Ottawa, territoire traditionnel et non cédé de la nation algonquine.

Les opinions exprimées dans ce report sont celles des auteurs et ne représentent pas nécessairement les opinions de la Société royale du Canada. 


\section{Contexte concernant la préparation de cette note de breffage}

Établi par le président de la Société royale du Canada en avril 2020, le Groupe de travail de la Société royale du Canada sur la COVID-19 s'est vu confier le mandat de dégager des perspectives éclairées par la recherche sur les grands enjeux sociétaux qui se posent au Canada relativement à sa réponse à la COVID-19 et à sa démarche subséquente de rétablissement.

Le Groupe de travail a établi un ensemble de sous-groupes de travail chargés de préparer rapidement des notes de breffage destinées à éclairer par des données probantes les réflexions des décideurs politiques.

\section{À propos des auteurs}

Timothy Caulfield, titulaire de la Chaire de recherche du Canada en droit et en politique de la santé, University of Alberta

Tania Bubela, professeure et doyenne, Faculté des sciences de la santé, Simon Fraser University Jonathan Kimmelman, professeur James McGill, unité d'éthique biomédicale, Université McGill Vardit Ravitsky, professeure agrégée, Département de médecine sociale et préventive, Université de Montréal

\section{Note des auteurs}

Nous tenons à remercier tous les membres du Groupe de travail de la Société royale du Canada sur la COVID-19 pour leur soutien et leurs commentaires, de même que Darren N. Wagner, pour ses éclairages et ses excellents conseils rédactionnels. Nous remercions également les pairs examinateurs anonymes qui ont contribué à ce texte par leurs suggestions et leurs commentaires judicieux.

Le présent rapport fait suite à l'article The COVID-19 pandemic will cause trust in science to be irreparably harmed, écrit par Timothy Caulfield et publié dans le Globe and Mail le 10 juillet 2020. 


\section{Table des matières}

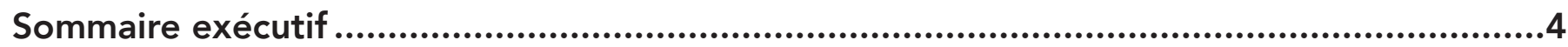

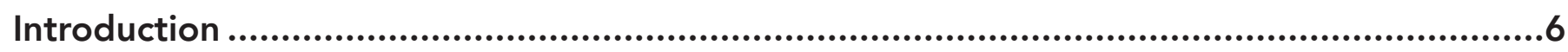

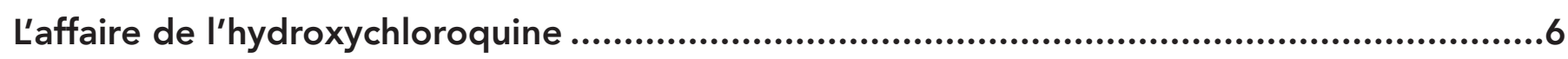

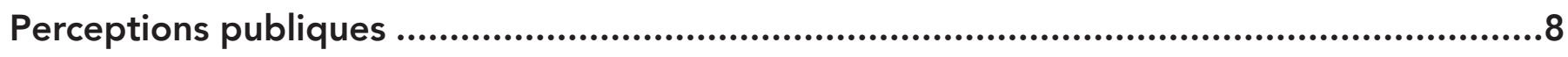

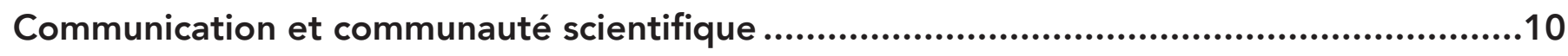

Politiques de santé publique et communication des travaux scientifiques ........................13

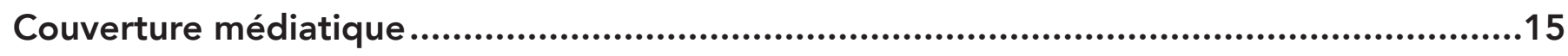

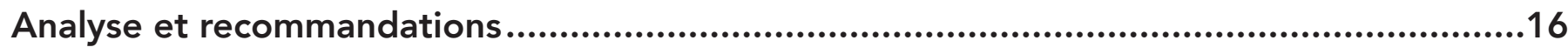

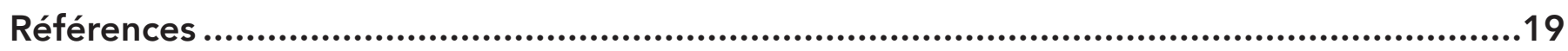


Les recherches scientifiques sur la COVID-19 sont à la fois menées et diffusées à une cadence effrénée. Bien qu'il soit inspirant de voir la communauté de la recherche répondre avec autant de vigueur à la crise causée par la pandémie, toute cette activité a par ailleurs engendré un chaos de mauvaises données, de résultats contradictoires et de manchettes exagérées. Alors que la polarisation, la déformation et la médiatisation des résultats scientifiques s'intensifient chaque jour, les inquiétudes se font de plus en plus sentir quant à la perspective que la science pertinente soit présentée au public d'une manière qui puisse causer de la confusion, créer de fausses attentes et éroder la confiance du public. Dans cette note, nous explorons les principaux enjeux associés à la présentation de la science dans le contexte de la pandémie de la COVID-19. Plusieurs de ces enjeux ne sont pas nouveaux. Mais la pandémie de la COVID-19 a braqué les projecteurs sur le processus de la recherche biomédicale et a amplifié les ramifications néfastes des problèmes

de communication publique. Nous devons faire mieux. À ce titre, nous conclurons ce rapport en formulant dix recommandations qui s'adressent aux acteurs clés qui interviennent dans la communication de la science sur la COVID-19, notamment les gouvernements, les bailleurs de fonds, les universités, les éditeurs, les médias et les communautés de recherche.

\section{Recommandations}

Nous formulons plusieurs recommandations qui, nous l'espérons, conserveront toute leur pertinence même après la présente pandémie.

1) La communauté de la recherche-y compris les organismes subventionnaires, les institutions et établissements de recherche, les comités d'éthique de la recherche, les chercheurs et les éditeurs - devraient préserver et défendre en priorité l'intégrité du processus de recherche. Les organismes fédéraux, provinciaux et institutionnels de financement de la recherche ainsi que les établissements de recherche, devraient examiner comment leurs critères, leurs mesures incitatives et leurs processus d'évaluation influencent la façon dont les travaux scientifiques sont représentés et communiqués au public.

2) Tout au long du processus de production et d'application du savoir, les chercheurs devraient présenter leurs travaux de façon mesurée, en situant leurs conclusions dans le contexte de l'ensemble des connaissances disponibles et en décrivant les limites, les forces et les faiblesses des méthodologies utilisées. Les organisations scientifiques pertinentes devraient envisager de faire de cette recommandation une obligation.

3) La transparence à l'égard des preuves, des données et des méthodes favorise une présentation mesurée et juste des constats scientifiques au public. Cette transparence exige que les chercheurs déposent leurs données et leurs résultats, surtout ceux tirés d'essais cliniques, dans des registres publiquement accessibles appropriés (p. ex., clinicaltrials.gov).

4) Les chercheurs devraient surveiller comment leurs travaux (et les travaux qui touchent leur domaine d'expertise) sont représentés dans la sphère publique et, lorsque cela s'impose, corriger les représentations erronées en employant un éventail de plateformes médiatiques, y compris les divers réseaux sociaux. La participation des chercheurs à des activités de communication devrait être soutenue, reconnue et encouragée. Les chercheurs devraient, au besoin, avoir accès à une formation appropriée à cet égard. 
5) La rigueur du processus de révision par les pairs devrait demeurer une priorité, peu importe les pressions externes exercées pour publier rapidement les résultats. La communauté de la recherche - dont les entités comme les IRSC, le CRSNG, le CRSH, le Conseil des académies canadiennes, etc. - devrait travailler étroitement avec les éditeurs scientifiques en vue d'élaborer des stratégies pour encadrer les examens par les pairs pendant les périodes de crise. Cela devrait être fait en considérant les moyens qui permettraient d'améliorer la viabilité du processus d'examen par les pairs, qui s'appuie actuellement sur le travail bénévole des chercheurs universitaires.

6) Une grande vigilance devrait être portée à la façon dont les résultats des recherches qui n'ont pas encore été examinées par des pairs - comme les préimpressions - sont représentés dans le domaine public. II est notamment important de souligner la nature préliminaire de ces résultats. De plus, les entités de financement de la recherche, les universités, les revues scientifiques, les associations scientifiques, etc., devraient réfléchir au rôle des préimpressions et à la façon de contrer leur influence néfaste potentielle sur le discours public.

7) Lorsque les établissements de recherche et les chercheurs produisent des communiqués de presse à l'intention du grand public, ils devraient s'abstenir d'exagérer les avantages ou les conséquences de leurs recherches, notamment des essais cliniques, situer leurs travaux dans le contexte des données probantes disponibles et accessibles, dont les résultats des essais cliniques, et décrire les limites des méthodologies utilisées. Dans le cadre du processus de communication, les chercheurs et les établissements de recherche devraient envisager de produire des résumés qui peuvent être compris par le grand public et par les groupes/ communautés pour qui les résultats des recherches peuvent être particulièrement pertinents.

8) Les organismes publics - tels que les autorités de santé publique et les organismes fédéraux de réglementation - devraient se montrer transparents au regard des données probantes (et autres considérations) utilisées pour éclairer leurs décisions, y compris en fournissant une évaluation honnête de l'état actuel des connaissances et de la nature évolutive de la science en période d'incertitude. Les organismes publics devraient aussi éviter de se montrer dogmatiques et devraient s'isoler de toute ingérence politique dans leur interprétation et leur présentation des résultats scientifiques.

9) Les médias d'information (et la presse populaire plus généralement) devraient s'efforcer de présenter les travaux scientifiques de manière aussi juste et instructive que possible, notamment en s'abstenant d'exagérer l'importance des résultats, de suggérer l'imminence de leur application ou d'extrapoler indûment les résultats au-delà de la portée de l'étude. Les journalistes devraient également situer les recherches dans le contexte de l'ensemble des données probantes disponibles et reconnaître, entre autres, les limites des méthodes particulières utilisées et la pertinence scientifique limitée des anecdotes, des témoignages et d'une étude en particulier.

10) Les chercheurs et les communicateurs scientifiques devraient toujours être conscients que les recherches sont susceptibles d'être interprétées d'une façon qui pourrait avoir, pour des personnes, des groupes ou des populations, des conséquences néfastes telles que des actes d'humiliation, de stigmatisation ou de racisme. Les communications devraient être faites en partenariat avec les participants aux recherches et leurs points de vue devraient être pris en compte tout au long du processus de recherche. 


\section{Introduction}

Depuis le début de l'année 2020, des dizaines de milliers d'articles de recherche examinés par des pairs et de préimpressions sur la COVID-19 ont été rendus publics (Coronavirus Research Publishing 2020). Le nombre d'articles présentés aux revues biomédicales prestigieuses a substantiellement augmenté, certaines revues recevant trois fois plus d'articles que d'ordinaire (Bauchner, Fontanarosa et Golub 2020).

Les recherches scientifiques sur la COVID-19 sont à la fois menées et diffusées à une cadence effrénée. À l'heure actuelle, le temps médian qui s'écoule entre la soumission d'un article à l'examen du comité de pairs d'une revue et l'acceptation de l'article n'est que de six jours (Palayew et al. 2020). II s'agit d'une augmentation époustouflante de la cadence d'acceptation des articles par rapport au délai approximatif de 100 jours qui avait cours d'avant la pandémie. Et certaines publications ont pu franchir l'étape de l'examen par les pairs en une journée seulement (Locher et al. 2020).

II est compréhensible que l'on souhaite que la science progresse rapidement pendant une pandémie (Gleick 2020). Mais bien qu'il soit inspirant de voir la communauté de la recherche répondre avec autant de vigueur à la crise causée par la pandémie, toute cette activité a par ailleurs contribué à amplifier l'environnement informationnel déjà passablement chaotique en introduisant de mauvaises données, des résultats contradictoires et des manchettes exagérées (Jaklevic 2020). Une journée, une étude publiée dans une revue biomédicale renommée est encensée comme constituant un ensemble irréfutable de données qui devrait (et ce fut le cas) guider nos actions et nos politiques (Sattui et al. 2020). Le jour suivant, la même étude est rétractée (Joseph 2020) (ou on demande qu'elle le soit) (Mandavilli 2020).

Alors que la polarisation, la déformation et la médiatisation des résultats scientifiques s'intensifient chaque jour, les inquiétudes se font de plus en plus sentir quant à la perspective que la science soit présentée au public d'une manière qui puisse causer de la confusion, créer de fausses attentes et éroder la confiance du public (Saitz et Schwitzer 2020).

Dans cette note, nous explorons certains des principaux enjeux associés à la présentation de la science dans le contexte de la pandémie de la COVID-19. Nous ne prétendons pas que cette note de breffage présente une analyse exhaustive de la façon dont la science est hiérarchisée, menée, encouragée et évaluée (Council of Canadian Academies 2010) - bien que nous évoquions tous ces sujets. Nous examinons plutôt certaines sources et incidences de la présentation problématique de la science sur la COVID-19 - y compris le risque qu'il y a de fragiliser la confiance du public et de compromettre la réussite des initiatives de santé publique. Plusieurs de ces enjeux ne sont pas nouveaux. Mais, comme nous le verrons ci-dessous, la pandémie de la COVID-19 a braqué les projecteurs sur le processus de la recherche en santé et a amplifié les ramifications néfastes des problèmes de communication publique.

\section{L'affaire de l'hydroxychloroquine}

Bien que l'on puisse citer de nombreux autres exemples de présentations moins qu'idéales de la science sur la COVID-19, la controverse de I'hydroxychloroquine illustre bien comment les choses 


\section{Sean Caulfield}

Distancing

Relief et jet d'encre sur papier de chiffon, $58 \times 58 \mathrm{~cm}, 2020$

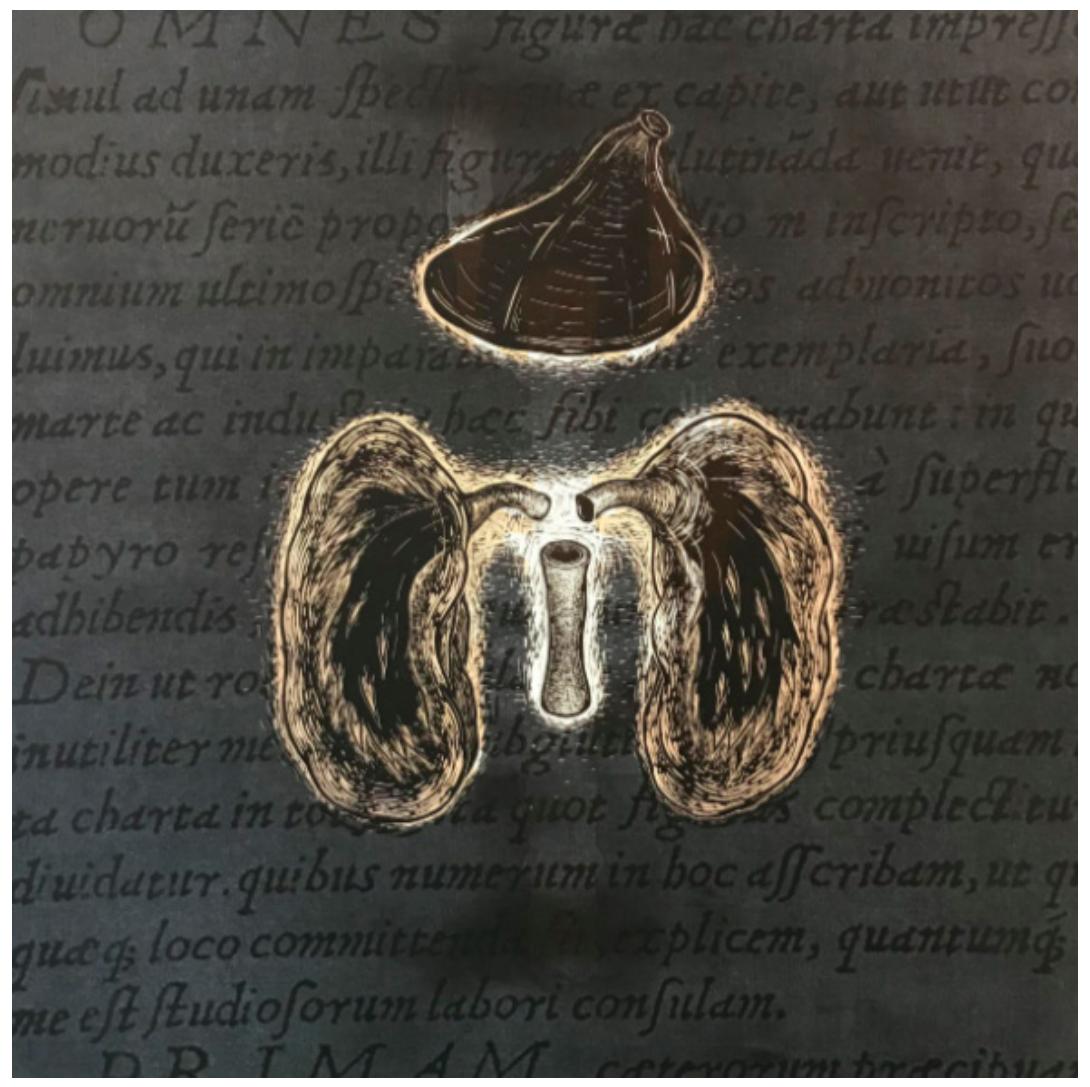

peuvent mal tourner et comment ce genre de bavure peut avoir une myriade de ramifications néfastes (Sattui et al. 2020).

À la mi-mars 2020, des chercheurs français ont publié une préimpression indiquant que I'hydroxychloroquine pourrait présenter certains avantages thérapeutiques pour le traitement de la COVID-19 (Gautret et al. 2020). II s'agissait d'une étude ouverte de petite envergure ( $N=80)$, qui avait été immédiatement critiquée pour ses carences méthodologiques, lesquelles auraient dû empêcher sa publication dans une revue arbitrée (Voss 2020). En effet, une analyse spécialisée réalisée ultérieurement a conclu que l'étude constituait « un manuscrit non instructif présentant de grossières lacunes méthodologiques » (Rosendaal 2020). Par suite directe de la publication de cette étude (qui, au moment de la rédaction du présent rapport, avait déjà malheureusement été citée plus de 1700 fois) et malgré les préoccupations scientifiques soulevées, I'hydroxychloroquine avait commencé à recevoir une attention favorable considérable dans les médias et son utilisation avait été prônée par des personnalités comme Elon Musk et Donald Trump. Tout cela a soulevé l'intérêt du public pour le médicament. Une étude a montré que le nombre de recherches sur Internet à son sujet est monté en flèche à la suite de ces appuis (M. Liu et al. 2020), tout comme le nombre de prescriptions hors indication du produit par des médecins (Vaduganathan et al. 2020), ce qui est encore plus troublant.

À mesure que se dessinait l'affaire de l'hydroxychloroquine (Thompson 2020) - et que le battage médiatique à son égard prenait de l'ampleur - de plus en plus de chercheurs dans le monde ont commencé à étudier le médicament, même si peu d'éléments indiquaient qu'il puisse avoir une utilité clinique substantielle (ou de tout ordre de grandeur) (University of Oxford - News 2020; Boulware et al. 2020; Kupferschmidt 2020; Skipper et al. 2020). Puis, à la mi-juin, une étude de 
grande envergure - publiée dans la revue influente The Lancet - a conclu que le médicament était susceptible de causer des événements indésirables graves. L'étude a suscité une réaction immédiate de la part de la communauté internationale de la recherche, dont certains essais cliniques ont même été temporairement interrompus en raison des craintes soulevées. Relevant des anomalies dans l'ensemble de données utilisé pour appuyer les conclusions sur les effets indésirables possibles du médicament, plusieurs autres scientifiques ont presque immédiatement critiqué l'étude et elle a rapidement fait l'objet d'une rétractation (Mahase 2020b).

L'affaire de l'hydroxychloroquine continue toujours d'évoluer (Gonsalves 2020). D'autres données publiées - fournies par des études observationnelles et des essais cliniques de valeur méthodologique variable - indiquent qu'il est peu probable que le médicament apporte un quelconque bienfait aux patients atteints de la COVID-19 (Qaseem et al. 2020). Par conséquent, vu le manque de données convaincantes, plusieurs essais cliniques importants ont été arrêtés, dont des essais financés par les NIH (Kiley 2020) et l'OMS (WHO 2020). De plus, les inquiétudes quant aux effets secondaires graves possibles du produit demeurent (Downes et al. 2020; U.S. Food and Drug Admin 2020).

Bien entendu, d'autres exemples de science de mauvaise qualité et de mauvaise communication des résultats scientifiques dans le contexte de la recherche sur la COVID-19 pourraient être cités (Day 2020; Schwitzer 2020). Mais la controverse de l'hydroxychloroquine - qui a pris de l'ampleur à cause de sa médiatisation et des appuis exprimés par des célébrités à une recherche préliminaire de qualité discutable - met en relief l'étendue des conséquences néfastes que peut avoir la piètre qualité des communications scientifiques, y compris l'introduction d'inefficacités dans les efforts de recherche sur la COVID-19 (p. ex. en nuisant au recrutement des participants à des essais cliniques bien conçus) (Ledford 2020), l'investissement de fonds publics dans des recherches discutables (Herper et Riglin 2020), la prise de mauvaises décisions au regard de l'allocation des ressources pharmaceutiques (Mahase 2020a), lesquelles peuvent mener à des pénuries de ressources (des pénuries d'hydroxychloroquine - qui ont potentiellement eu des conséquences pour des personnes atteintes de troubles immunitaires chroniques - ont été signalées dans la plupart des provinces canadiennes) (Mendel et al. 2020), l'incitation à prescrire des traitements inutiles et potentiellement nuisibles (Lovelace Jr. 2020) et la création d'attentes trop élevées et sans fondement chez le public (p. ex., $23 \%$ des Canadiens - et $30 \%$ de Québécois - croient à tort que le médicament est efficace) (Everts et Greenberg 2020). Le battage médiatique initial et la rétractation subséquente des résultats de recherche ont alimenté des théories conspirationnistes (Mikkelson 2020), ont servi à polariser le discours public et, ce qui pose sans doute le plus problème, ont peut-être contribué à diminuer la confiance du public envers la science (Laurent 2020).

Malgré les données qui démontrent de plus en plus que le produit n'est pas efficace, certaines personnes continuent de croire aux bienfaits de l'hydroxychloroquine - en partie parce qu'elle est devenue associée à une certaine position idéologique de par son lien avec des personnalités politiques très en vue comme le président Trump (Dearment 2020). Lorsqu'une croyance devient un élément de l'identité d'une personne, il peut être très difficile de lui faire changer d'avis (Kaplan, Gimbel et Harris 2016).

\section{Perceptions publiques}

Instaurer et préserver la confiance du public - y compris dans la science et les institutions scientifiques - est particulièrement essentiel pendant une pandémie (Balog-Way et McComas 
2020; Udow-Phillips et Lantz 2020). Par exemple, certaines données (bien que de nature observationnelle) associent la confiance dans les institutions pertinentes avec la probabilité d'adopter les comportements préventifs requis (Devine et al. 2020; Fukuyama 2020; Goldberg et al. 2020; Lep, Babnik et Hacin Beyazoglu 2020). Une analyse réalisée en France, par exemple, a conclu que « les régions qui font confiance aux autorités diminuent davantage leurs déplacements liés à des activités non nécessaires que les régions où la confiance est plus faible " (Bargain et Aminjonov 2020). Les constats de cette étude concordent avec ceux d'une recherche australienne qui associe la confiance dans le gouvernement et les autorités de santé à une plus large adoption des comportements de distanciation physique et d'hygiène recommandés (Seale et al. 2020). Une autre recherche, de l'Université Johns Hopkins, a précisément examiné la question de la méfiance à l'égard de la science et a conclu qu'elle était fortement associée au refus d'adopter des stratégies préventives - comme la distanciation physique - et, sans surprise, d'écouter les conseils des experts de la santé publique sur la COVID-19 (Barry, Han et McGinty 2020). La méfiance envers les institutions pertinentes a également été associée à une croyance accrue aux mythes et aux théories conspirationnistes sur la COVID-19 (Pickles et al. 2020).

Le public canadien suit de très près les événements qui entourent la pandémie. Un sondage mené en juin 2020 par l'Université Carleton, par exemple, a révélé que $82 \%$ des Canadiens suivaient les nouvelles sur la COVID-19 « chaque jour » (53 \%) ou « la plupart des jours (29\%) (Greenberg et Everts 2020). Cela signifie que le public est témoin du processus parfois tumultueux de l'élaboration des connaissances scientifiques (qui peut comprendre des faux pas) et de la nature changeante du consensus scientifique. Et il remarque les manchettes sensationnalistes, les rétractations et les désaccords qui opposent les différents experts scientifiques. Il est donc à craindre que les nombreuses présentations boiteuses des résultats scientifiques minent la confiance du public (Drage O'Reilly 2020), en particulier à mesure que la pandémie se prolonge et que le sentiment mondial dominant évolue, comme certaines recherches semblent l'indiquer, de la peur à la frustration et à la colère (Lwin et al. 2020).

Une étude réalisée récemment par la London School of Economics indique que la crise de la COVID-19 pourrait avoir eu une influence défavorable sur la perception des gens à l'égard des scientifiques, notablement chez les personnes qui ont peu ou pas du tout de formation scientifique (Aksoy, Eichengreen et Saka 2020a). Plus précisément, les chercheurs ont conclu - en s'appuyant sur les pandémies précédentes - que la crise de la COVID-19 « érodera la confiance accordée aux différents scientifiques, le degré d'honnêteté qui leur est prêté et le sentiment que leurs activités profitent au public » (Aksoy, Eichengreen et Saka 2020a). Une autre étude, un sondage français, a calculé que le degré de confiance dans la science avait diminué de $10 \%$ - principalement en raison de la frustration suscitée par deux sujets polarisants : la débâcle de l'hydroxychloroquine et les volte-face des autorités concernant le port du masque (Matthew 2020).

Jusqu'à maintenant, le discours public au Canada sur la COVID-19 n'a pas suscité autant de division que ce qu'on a pu observer aux États-Unis et dans certains autres pays (Merkley et al. 2020; Padilla et Hípola 2020). La confiance à l'égard de nos institutions sanitaires et scientifiques demeure relativement forte (Statistics Canada 2020). La majorité des Canadiens diraient qu'ils ont confiance dans les chercheurs universitaires, les professionnels de la santé et les autorités publiques de santé (Statistics Canada 2020). Mais nous ne devons pas nous asseoir sur nos lauriers pour autant (Robinson 2020). Nous avions déjà quelques indications - avant la pandémie - que la confiance envers la science avait commencé à fléchir et que de nombreux Canadiens considéraient 
que la communauté scientifique était élitiste (Ontario Science Centre 2017; Semeniuk 2018; Weber 2019). Selon d'autres recherches, la pandémie pourrait conduire à une érosion générale et durable de la confiance dans les institutions publiques (Aksoy, Eichengreen et Saka 2020b). II y a aussi certains enjeux complexes de confiance que l'on doit considérer dans le contexte de populations particulières - notamment celles, comme les populations Autochtones (Kolopenuk 2020; Government of Canada 2019), qui ont été mal desservies, voire lésées par les institutions de recherche existantes.

La confiance peut se perdre rapidement et entraîner dans sa chute des conséquences désastreuses (Robinson 2020). En effet, la perception du public à l'égard de la science et les problèmes de confiance sont susceptibles d'occuper une place encore plus proéminente lorsque viendra le temps de faire vacciner la population. Des études ont conclu qu'une méfiance envers la science est associée à une intention diminuée de recevoir un vaccin contre la COVID-19 (Palamenghi et al. 2020). Plusieurs au Canada ont des craintes par rapport à tout vaccin et cette hésitation ne fait qu'augmenter. En août 2020, un sondage a révélé que seulement 46 \% des Canadiens disaient qu'ils « accepteraient de recevoir un vaccin dès qu'il y en aurait un de disponible » et trois personnes sur cinq avaient des soucis quant à l'innocuité d'un tel vaccin (Angus Reid Institute 2020). Les prétentions des groupes opposés aux vaccins ont manifestement eu une certaine influence sur la perception du public, entre autres parce qu'elles s'appuient (et alimentent) les craintes au sujet de la qualité des données scientifiques (Crow et Stacey 2020). Considérant les préoccupations que soulève la possibilité d'une ingérence politique dans le processus de recherche d'un vaccin (LaFraniere et al. 2020) - particulièrement aux États-Unis - les problèmes sociaux et sanitaires associés à l'érosion de la confiance dans les institutions scientifiques et les décisions éclairées par la science sont susceptibles de s'amplifier. D'ailleurs, un sondage mené en août 2020 a révélé que « $78 \%$ des Américains craignent que le processus d'approbation d'un éventuel vaccin contre la COVID-19 soit davantage influencé par des motifs politiques que par la science » (Silverman 2020).

\section{Communication et communauté scientifique}

Évidemment, pour présenter de manière exacte, équilibrée et digne de confiance les travaux scientifiques, il est au départ essentiel de faire en sorte que ces travaux soient menés correctement et de façon transparente, ce qui exige notamment que le public puisse avoir accès aux données pertinentes (p. ex., en mettant en place des registres de données publiquement accessibles). Des préoccupations grandissantes s'élèvent concernant la possibilité que les pressions associées à la production et à la diffusion des travaux scientifiques sur la COVID-19 aient un effet négatif sur le plan de la qualité (Dinis-Oliveira 2020). Un des meilleurs moyens de créer de la confusion et de perdre la confiance du public est de publier et de promouvoir des recherches de mauvaise qualité, négligentes ou, pire encore, frauduleuses (Yarborough 2014). Malheureusement, cela se produit trop souvent en cette ère de publication effrénée (Blaming 2020; Steinberg 2020) - qui, comme un commentateur a suggéré, a créé « un déluge de recherches de piètre qualité » qui sont en train de "saboter la mise en œuvre d'une réponse efficace et fondée sur des données probantes" (Glasziou, Sanders et Hoffmann 2020). Cela comprend les rétractations très médiatisées et fréquentes d'articles de revues à comité de lecture (Yeo-Teh et Tang 2020), bien que l'on ne sache pas encore précisément dans quelle mesure le taux de rétractation soit inhabituel, ou même s'il l'est un tant soit peu (Abritis, Marcus et Oransky 2020). (Au moment de la rédaction de la présente 
note, Retraction Watch, une entité qui surveille ce type d'activité, a relevé 36 études rétractées sur la COVID-19.)

Bien qu'une analyse détaillée des institutions de recherche et des structures incitatives existantes dépasse la portée du présent rapport, il paraît évident que maintenir l'intégrité du processus de recherche devrait être une priorité. Pendant une pandémie, il y a un sentiment d'urgence qui est tout à fait compréhensible (Tingley 2020). Mais on ne pas devrait permettre que la volonté d'arriver à des résultats rapides érode les normes scientifiques (Pang et Elkhodiry 2020). Comme Alex John London et Jonathan Kimmelman ont succinctement rappelé, " les crises ne doivent pas servir d'excuse à un rabaissement des normes scientifiques » (London et Kimmelman 2020). Ce point de vue a été secondé par H. Clifford Lane et Anthony Fauci : "Les recherches cliniques robustes sur le plan scientifique et éthique demeurent la voie la plus rapide et la plus efficace pour trouver des traitements et des stratégies de prévention efficaces pour les patients atteints de la COVID-19 „ (Lane et Fauci 2020). Malheureusement, certaines indications laissent supposer qu'une partie importante des recherches actuellement menées - selon une analyse des essais cliniques enregistrés (ECE) - est susceptible de ne produire qu'un « faible niveau de données probantes », puisque peu d'ECE sont de qualité et que, par conséquent « la plupart des études ne généreront pas de données scientifiques de grande valeur » (Di Girolamo et Meursinge Reynders 2020; Pundi et al. 2020).

La quête de la vitesse peut également rendre problématique la façon dont les travaux scientifiques sont publiés et présentés au public et aux décideurs. Certains ont évoqué la possibilité, par exemple, que l'énorme volume d'articles que doivent examiner les comités de lecture soit en train de submerger le processus d'examen - comme en témoigne l'article qui a lancé la controverse entourant l'hydroxychloroquine (Locher et al. 2020) - et que des « constats mal étayés ou même inexacts soient diffusés, amplifiés et potentiellement incorporés dans le discours scientifique et populaire » (Bell et Green 2020). Certains de ces travaux ont été rapidement rétractés (Retracted coronavirus (COVID-19) papers), mais une fois que des études sont diffusées dans la presse populaire et dans les médias sociaux, il est difficile de réparer les dégâts - y compris les dommages causés à la confiance du public (Wysong 2020).

Au vu de ces considérations, des recommandations ont été faites pour améliorer le processus de publication et d'examen par les pairs (Bauchner, Fontanarosa et Golub 2020), comme celles d'établir de nouvelles normes éditoriales, pour préserver la qualité des écrits pendant les urgences de santé publique, et d'exiger que les examinateurs soient bien formés (Bazdaric et Smart 2020; Palayew et al. 2020). Certaines revues - dont The Lancet, la publication impliquée dans une des rétractations notoires récentes liées à la COVID-19 (Medical Xpress 2020; Rabin 2020) - ont déjà commencé à suggérer que le processus d'examen par les pairs soit revu pour garantir un contrôle plus minutieux des méthodes et données des études examinées (Caulfield 2020).

Mais il faudraitégalement s'attaquer au problème de l'explosion des préimpressions, des recherches qui sont mises en circulation avant d'avoir été examinées par un comité de pairs (Van Schalkwyk et al. 2020). Les préimpressions peuvent constituer un outil précieux pour diffuser des données et pour obtenir des critiques constructives de la part de collègues. (La plupart des revues, dont 80 \% des publications à impact élevé, permettent la circulation des préimpressions avant la soumission des articles à l'examen des pairs (Massey et al. 2020)). Mais les préimpressions peuvent aussi donner lieu à la circulation de recherches non vérifiées et douteuses et venir brouiller le discours public (une préimpression est justement à l'origine de l'affaire de l'hydroxychloroquine). Elles

Faisons mieux les choses : représentation publique de la science sur la COVID-19 
sont produites et consultées à une cadence extrêmement élevée pendant la pandémie. Et elles ont une influence sur le discours public et politique (Majumder et Mandl 2020). Selon une étude (ironiquement une préimpression portant sur les préimpressions), la pandémie aurait entraîné une consultation accrue des préimpressions par les chercheurs, le public et les médias. Par exemple, les auteurs de l'étude ont constaté que " les préimpressions sur la COVID-19 étaient consultées et diffusées au moins 15 fois plus que celles qui ne concernaient pas la COVID-19 " (Fraser et al. 2020). Considérant cet état de fait, certains chercheurs ont décidé de produire des analyses informelles rapides et publiquement accessibles des préimpressions en circulation pour éclairer le public et les responsables des politiques (Eisen et Tibshirani 2020).

La façon dont les scientifiques communiquent leurs travaux dans les médias, dans les médias sociaux et directement au public, doit également être examinée. La façon dont les travaux sont communiqués sur les réseaux sociaux, par exemple, peut conditionner les citations subséquentes, mais aussi leur traitement dans le discours public et politique (Kousha et Thelwall 2020). Les membres de la communauté scientifique sont de plus en plus incités à présenter les résultats de leurs travaux en des termes enthousiastes. En effet, des pressions et des incitations se font sentir à toutes les étapes du processus de création du savoir pour encourager les chercheurs à mousser la valeur de leurs travaux (Bubela 2006; Bubela et al. 2009; Caulfield et Condit 2012), que ce soit lors de la présentation des demandes de subventions (Matthews 2016), de la rédaction des résultats (Vinkers, Tijdink et Otte 2015), de la production des communiqués de presse des

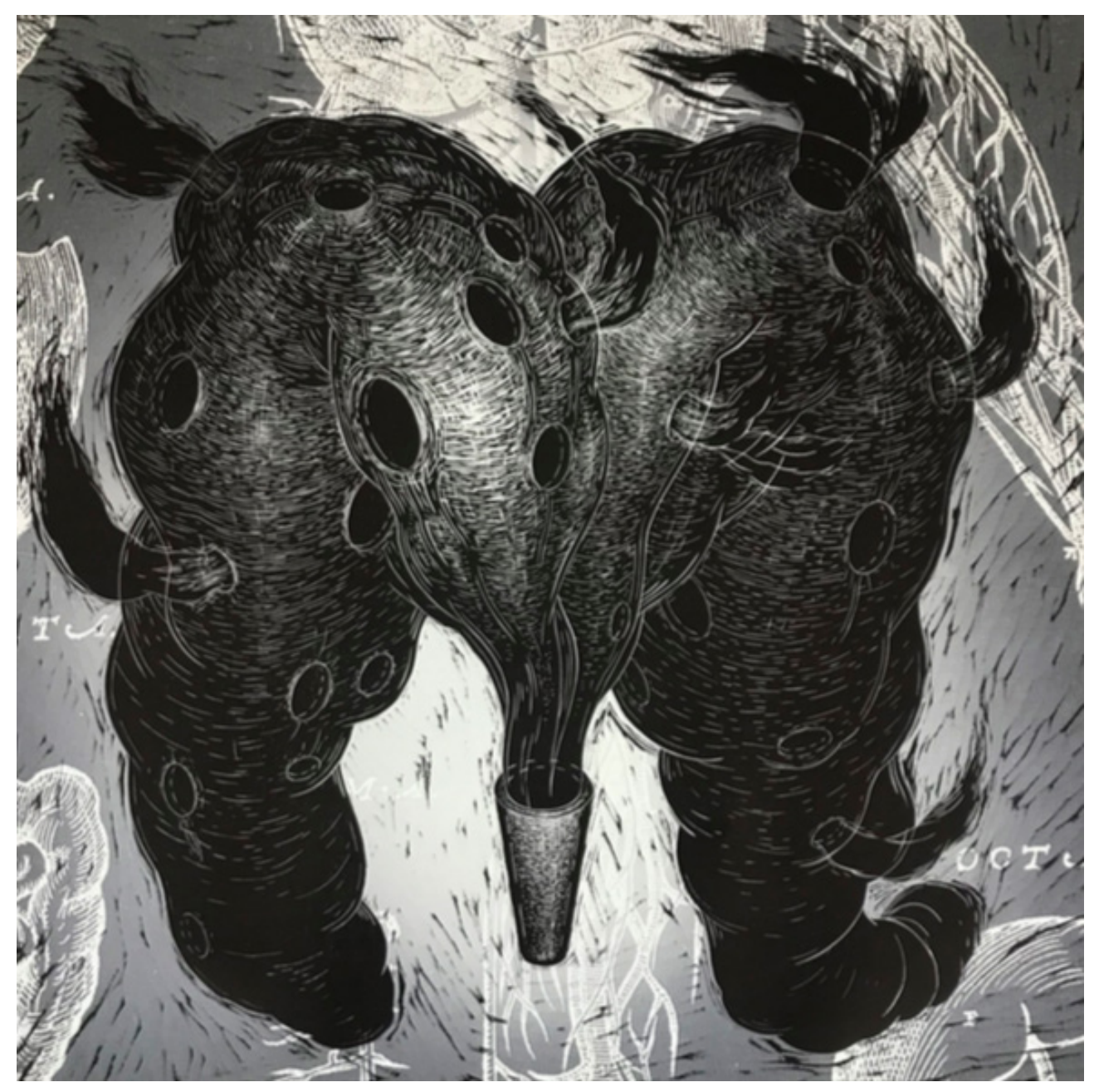

Sean Caulfield

Gravure sans titre tirée du livre d'artiste Infodemic

Relief et jet d'encre, $58 \times 58 \mathrm{~cm}, 2020$ 
établissements de recherche (Yavchitz et al. 2012) ou des interactions avec la presse populaire (Kamenova et Caulfield 2015). Et comme nous l'avons vu dans d'autres domaines, toute cette hyperbolisation (Ball 2015) peut avoir un effet profond sur la compréhension du public, sur les politiques scientifiques et sanitaires (Caulfield 2018), sur la mise en marché des produits et des thérapies associés (Caulfield et al. 2016) et, peut-être (Master et Resnik 2013), sur la confiance du public (Resnick 2019).

Il est essentiel que la communauté scientifique continue de participer à la conversation publique, y compris pour remettre en cause les fausses représentations et la propagande utilisées pour polariser les perceptions publiques. Mais il est également essentiel que les membres de la communauté de la recherche présentent leurs travaux de façon mesurée et exacte (Leeming 2018), y compris en expliquant les limites de leurs travaux et la place qu'ils occupent dans le corpus plus large des connaissances.

Inversement, il est également important qu'un large éventail de communautés participent à la conversation scientifique, surtout lorsqu'il s'agit de recherches qui influenceront les interventions publiques sanitaires (Tworek, Beacock et Ojo 2020). Ces interventions ont des conséquences à la fois souhaitables et indésirables, et les fardeaux économiques, sociaux et sanitaires ne sont pas également répartis. Dans le contexte de la COVID-19, les données laissent penser que certaines communautés supportent un fardeau de maladie plus lourd que d'autres et, en même temps, ont un niveau de méfiance plus élevé à l'égard, par exemple, du processus de recherche d'un vaccin (Hoffman 2020). Les meilleures pratiques de recherche dans les domaines des sciences sociales et de la santé intègrent de plus en plus l'avis des partenaires communautaires et des patients, que ce soit au stade de la gestation des questions de recherche, de la conception méthodologique, de la réalisation des recherches ou de l'interprétation, de la circulation et de la communication des résultats. Ce dernier point devient impératif lorsque les constatations scientifiques sont susceptibles d'être interprétées d'une manière qui pourrait encourager la stigmatisation ou les comportements ouvertement racistes envers certaines personnes, communautés ou populations. Cette philosophie de la participation du public est clairement exprimée dans les textes fédéraux Énoncé de politique des trois conseils : Éthique de la recherche avec des êtres humains (TriCouncil Policy Statement 2018) et Stratégie de recherche axée sur le patient (Strategy 2019). La nécessité reconnue d'une telle participation est fondée sur la prémisse voulant que la confiance du public soit meilleure si ceux et celles qui seront les plus touchés par la recherche participent activement à la recherche. Bien que ce sujet dépasse la portée du présent article, les pratiques de recherche dans le domaine de la santé des Autochtones vont encore plus loin dans ce sens : les recherches sur les Autochtones sont de plus en plus menées et contrôlées par les communautés autochtones (The First Nations Principles of OCAP).

\section{Politiques de santé publique et communication des travaux scientifiques}

Les autorités de santé publique - régionales, nationales et internationales - représentent une source essentielle d'information pendant une pandémie. Bien qu'un degré relatif de controverse ait entouré certaines recommandations émises par des entités comme l'Organisation mondiale de la Santé, les Centers for Disease Control et Prevention (CDC), aux États-Unis, et l'Agence de la santé publique du Canada (ASPC), les cliniciens, les établissements de soins de santé, les éducateurs, le public et les politiciens se tournent vers les autorités sanitaires pour recevoir des mises à jour sur les données les plus récentes et des recommandations sur la meilleure marche à 
suivre (Carleton 2020; Goldberg et al. 2020). Par conséquent, il est d'une importance capitale que les résultats scientifiques soient communiqués d'une manière qui préserve la confiance du public dans la science et les institutions pertinentes.

Les autorités de santé publique devraient, par exemple, expliquer de manière honnête et claire l'état des connaissances qui sont utilisées pour étayer les recommandations (Leask 2020; Mello, Greene et Sharfstein 2020; Robinson 2020). Elles devraient notamment « décrire de manière transparente et ouverte ce que l'on sait et ce que l'on ne sait pas sur le virus SRAS-CoV-2 et la maladie à COVID-19 " (Pak et Adegboye 2020). Les jugements sans fondement ou simplistes sur certains avantages ou inconvénients - aussi noble soit l'objectif - ne servent qu'à polariser davantage le processus qui, avec le temps, sera susceptible de miner la confiance et la perception du public à l'égard de la science et des scientifiques. Il faut aussi prêter attention au mode de communication - les médias visuels se distinguant des sources imprimées, puisqu'ils nécessitent des porte-paroles, un environnement et un message cohérent pour assurer la clarté de l'information et préserver la confiance du public (Luth, Jardine et Bubela 2013).

Comme nous l'avons vu, les recommandations changeantes sur le port du masque (Zhang et al. 2020) en public ont été pointées du doigt comme sources potentielles de la méfiance du public (Urback 2020). Des commentateurs ont affirmé que cette évolution dans les directives - ou la "volte-face ", comme l'ont étiquetée les personnes qui sont critiques à l'égard des politiques sur le port du masque (Toronto Sun 2020) - a contribué à diminuer la confiance du public envers les autorités de la santé (Gerson 2020). Dans de telles situations, les autorités de santé publique ne devraient pas hésiter à parler avec franchise de la nature équivoque et changeante des données scientifiques. Comme l'ont relevé les experts de la santé publique Rutter, Wolert et Greenhalph, "la plupart des données sont imparfaites ou incomplètes " pendant une pandémie et nous devons " être honnêtes et transparents à ce sujet » (Rutter, Wolpert et Greenhalgh 2020). Bien entendu, le processus scientifique se déroule presque toujours de cette façon. La situation actuelle n'est pas propre à la pandémie que nous vivons. Par conséquent, il est également très important que le public comprenne la nature de la recherche et du processus d'application des connaissances scientifiques.

Pendant une pandémie, les décisions liées à la santé publique doivent souvent être prises sur la base d'un ensemble imparfait de données (Greenhalgh 2020). Et toute recommandation basée sur une science en émergence est appelée à évoluer. Réviser une position lorsque de nouvelles informations sont disponibles et/ou les conditions sociales changent ne devrait pas être vu comme une défaillance du système (Dupré 2020). Bien qu'il soit compréhensible que les autorités de santé publique puissent être tentées de présenter un message ferme et sans équivoque, il est important qu'elles soient explicites quant aux incertitudes qui entourent les données. En effet, certaines indications laissent croire que se montrer transparent à propos des incertitudes peut en réalité avoir un effet positif sur la crédibilité (Ratcliff et al. 2018), la confiance (Fleerackers 2020) et la compréhension du public (Jensen et al. 2011; Porter 2020). Les autorités de santé publique peuvent transmettre un message clair et concret, qui mobilise nos valeurs communes, mais qui reflète tout de même les connaissances scientifiques les plus récentes. Comme l'a souligné Dominique Brossard, un expert des communications scientifiques, " en fin de compte, il est mieux de dire: "La meilleure pratique à adopter est la suivante; nous n'en sommes pas totalement certains, mais nous vous tiendrons au courant dès que nous en saurons davantage" " (Drage O'Reilly 2020). 
Certains ont suggéré qu'il est important de préparer le public en lui expliquant, preuves à l'appui, pourquoi des stratégies de prévention supplémentaires pourraient devoir être mises en œuvre. Des détails pourraient être fournis, par exemple, concernant les données probantes réunies et les objectifs des stratégies envisagées (Seale et al. 2020). Et les autorités de santé publique doivent aussi utiliser un large éventail de plateformes de communication, particulièrement les médias sociaux, pour garantir que les messages basés sur la science occupent une place de premier plan dans le discours public (Lovari 2020). Il faudrait pour cela notamment travailler avec les plateformes des réseaux sociaux pour assurer un bon classement des « liens qui renvoient vers les recommandations des autorités de santé reconnues " (Limaye et al. 2020).

La question sans doute la plus troublante actuellement est celle de l'influence politique. Les décisions des institutions de santé fondées sur la science doivent être prises de manière indépendante et sans interférence politique. Faire autrement pourrait compromettre grandement la capacité de ces institutions à contribuer à la santé publique. Les mesures prises récemment aux États-Unis par la FDA (p. ex. le message véhiculé et l'approbation douteuse d'un traitement au plasma de convalescent) (Kupferschmidt et Cohen 2020; McGinley et al. 2020) et les CDC (p. ex., le renversement de la politique sur le dépistage des personnes asymptomatiques) (Sheridan 2020; Troisi 2020) rappellent comment l'ingérence politique peut avoir une influence à la fois sur la confiance du public et sur la façon dont la science est représentée (Wilson 2020).

\section{Couverture médiatique}

La façon dont les médias couvrent la science, surtout pendant une pandémie (Gozzi et al. 2020; Q. Liu et al. 2020), est également importante. Elle peut influer sur les perceptions et les attitudes du public (Zheng, Goh et Wen 2020), l'élaboration des politiques, les pratiques cliniques et les priorités de recherche. Et la couverture de l'actualité peut favoriser la diffusion de fausses informations et la polarisation du discours public (Green et al. 2020).

Les personnes qui travaillent dans la presse populaire - que ce soit à la télévision, à la radio, dans les médias imprimés ou en ligne - devraient éviter de surfaire ou de déformer les nouvelles scientifiques, y compris le caractère certain d'un résultat (Abbas et Lamb 2020; Strazewski 2020). Les percées scientifiques qui peuvent véritablement changer la donne sont extrêmement rares (par exemple, moins de $10 \%$ des médicaments expérimentaux suffisamment prometteurs pour faire l'objet d'essais cliniques seront approuvés pour un usage clinique) (Lowe 2019). La réalité est que la recherche scientifique est un processus itératif et, en général, long. Les médias, toutefois, préfèrent les assertions définitives d'une efficacité prochaine.

De nombreuses organisations journalistiques ont souligné à quel point il est important que les reportages soient fidèles à la réalité et mesurés (Coronavirus: Resources for Reporters 2020; Journalists' Resources 2020; Hanage et Lipsitch 2020; Mulcahey 2020). Pourtant, comme en témoigne l'affaire de l'hydroxychloroquine, une bonne partie des informations relayées par les journalistes ont été moins qu'idéales et les ramifications de ces lacunes sont loin d'être négligeables. Comme les experts de la communication scientifique Saitz et Schwitzer ont relevé dans leur analyse, les médias d'information se concentrent trop souvent sur une seule étude (et s'emballent pour elle) et/ou accordent une trop grande importance aux implications possibles d'un résultat sans situer la recherche dans le contexte des autres études disponibles (Saitz et Schwitzer 2020). 
Certains ont aussi soutenu que le contenu couvert par les médias, surtout dans les premiers jours de la pandémie, posait problème. Une étude, par exemple, a trouvé que la couverture des informations à la télévision portait principalement sur les décès et les taux de décès et très peu sur les comportement préventifs possibles (Basch et al. 2020).

Les médias d'information pourraient certainement améliorer leurs pratiques, mais il ne faudrait pas oublier qu'une bonne part des informations erronées et de l'hyperbolisation observées dans la presse viennent des chercheurs et des institutions de recherche eux-mêmes (Caulfield et Condit 2012; Woolston 2014). Il y a une relation entre la façon dont la recherche est présentée, par exemple, dans les communiqués de presse - qui moussent souvent les résultats des recherches - et la façon dont la science est présentée au public. Bien entendu, on retrouve une grande part de la désinformation qui circule concernant les travaux scientifiques sur la COVID-19 sur les réseaux sociaux. Elle est créée et véhiculée non pas par des journalistes professionnels, mais par les utilisateurs des plateformes de réseaux sociaux. En effet, on a déterminé que les médias sociaux constituaient la principale source de désinformation sur la COVID-19 (et que ceux qui tiraient leurs informations sur les médias sociaux étaient plus susceptibles de croire aux fausses informations) (Caulfield 2020; Bridgman et al. 2020). La presse populaire demeure toutefois une source importante d'information sur la pandémie et peut avoir une grande influence sur la façon dont la science est perçue et utilisée.

\section{Analyse et recommandations}

La science subit depuis toujours diverses pressions externes, dont les prescriptions idéologiques (Baran, Goldman et Zelikova 2019), les exigences militaires et de défense nationale (Finkbeiner 2018) ainsi que la sempiternelle quête du profit. Et bien entendu, les structures incitatives inhérentes au monde de la recherche - le fait par exemple de récompenser la quantité et les "facteurs d'impact » davantage que la qualité et les bienfaits pour la société (Plackett 2020) conditionnent elles aussi, pour le meilleur et pour le pire, le processus de recherche. Les décisions politiques prises par les gouvernements, les organismes subventionnaires et les établissements de recherche au regard de la façon de soutenir et de financer la recherche - y compris celle de la promotion de la commercialisation qui a été adoptée par la plupart des entités canadiennes de financement de la recherche (Caulfield et Ogbogu 2015) - contribuent à déterminer comment la recherche est menée et présentée au public.

Nous reconnaissons qu'une réflexion approfondie sur les facteurs complexes, interdépendants et systémiques qui conditionnent la perception publique de la science s'impose probablement. Nous reconnaissons également que l'amélioration seule de la représentation de la science n'aboutira pas nécessairement à de meilleures politiques et à un public mieux informé. Ces questions sont complexes et difficiles à résoudre. Notre objectif, toutefois, est plus restreint : notre attention se porte principalement les principaux responsables de la communication de la science sur la COVID-19. La façon dont les travaux scientifiques ont été rapportés pendant la présente pandémie a influencé la perception du public, les politiques sanitaires et scientifiques ainsi que l'adoption des stratégies de prévention. Mais les problèmes de communication survenus au cours de la crise sanitaire publique actuelle ne datent pas d'aujourd'hui. En effet, à maints égards, ils ont contribué à mettre en lumière les effets néfastes de plusieurs lacunes qui compromettent depuis longtemps la façon dont la science est communiquée, y compris les questions reliées à l'interprétation des résultats de recherche, au processus de publication, à l'élaboration des communiqués de presse 
et à la couverture médiatique. En conséquence, nous formulons plusieurs recommandations qui, nous l'espérons, conserveront toute leur pertinence même après la présente pandémie.

1) La communauté de la recherche-y compris les organismes subventionnaires, les institutions et établissements de recherche, les comités d'éthique de la recherche, les chercheurs et les éditeurs - devraient préserver et défendre en priorité l'intégrité du processus de recherche. Les organismes fédéraux, provinciaux et institutionnels de financement de la recherche ainsi que les établissements de recherche, devraient examiner comment leurs critères, leurs mesures incitatives et leurs processus d'évaluation influencent la façon dont les travaux scientifiques sont représentés et communiqués au public.

2) Tout au long du processus de production et d'application du savoir, les chercheurs devraient présenter leurs travaux de façon mesurée, en situant leurs conclusions dans le contexte de l'ensemble des connaissances disponibles et en décrivant les limites, les forces et les faiblesses des méthodologies utilisées. Les organisations scientifiques pertinentes devraient envisager de faire de cette recommandation une obligation.

3) La transparence à l'égard des preuves, des données et des méthodes favorise une présentation mesurée et juste des constats scientifiques au public. Cette transparence exige que les chercheurs déposent leurs données et leurs résultats, surtout ceux tirés d'essais cliniques, dans des registres publiquement accessibles appropriés (p. ex., clinicaltrials.gov).

4) Les chercheurs devraient surveiller comment leurs travaux (et les travaux qui touchent leur domaine d'expertise) sont représentés dans la sphère publique et, lorsque cela s'impose, corriger les représentations erronées en employant un éventail de plateformes médiatiques, y compris les divers réseaux sociaux. La participation des chercheurs à des activités de communication devrait être soutenue, reconnue et encouragée. Les chercheurs devraient, au besoin, avoir accès à une formation appropriée à cet égard.

5) La rigueur du processus de révision par les pairs devrait demeurer une priorité, peu importe les pressions externes exercées pour publier rapidement les résultats. La communauté de la recherche - dont les entités comme les IRSC, le CRSNG, le CRSH, le Conseil des académies canadiennes, etc. - devrait travailler étroitement avec les éditeurs scientifiques en vue d'élaborer des stratégies pour encadrer les examens par les pairs pendant les périodes de crise. Cela devrait être fait en considérant les moyens qui permettraient d'améliorer la viabilité du processus d'examen par les pairs, qui s'appuie actuellement sur le travail bénévole des chercheurs universitaires.

6) Une grande vigilance devrait être portée à la façon dont les résultats des recherches qui n'ont pas encore été examinées par des pairs - comme les préimpressions - sont représentés dans le domaine public. II est notamment important de souligner la nature préliminaire de ces résultats. De plus, les entités de financement de la recherche, les universités, les revues scientifiques, les associations scientifiques, etc., devraient réfléchir au rôle des préimpressions et à la façon de contrer leur influence néfaste potentielle sur le discours public.

7) Lorsque les établissements de recherche et les chercheurs produisent des communiqués de presse à l'intention du grand public, ils devraient s'abstenir d'exagérer les avantages ou les conséquences de leurs recherches, notamment des essais cliniques, situer leurs travaux dans le contexte des données probantes disponibles et accessibles, dont les résultats des essais cliniques, et décrire les limites des méthodologies utilisées. Dans le cadre du processus

Faisons mieux les choses : représentation publique de la science sur la COVID-19 
de communication, les chercheurs et les établissements de recherche devraient envisager de produire des résumés qui peuvent être compris par le grand public et par les groupes/ communautés pour qui les résultats des recherches peuvent être particulièrement pertinents.

8) Les organismes publics - tels que les autorités de santé publique et les organismes fédéraux de réglementation - devraient se montrer transparents au regard des données probantes (et autres considérations) utilisées pour éclairer leurs décisions, y compris en fournissant une évaluation honnête de l'état actuel des connaissances et de la nature évolutive de la science en période d'incertitude. Les organismes publics devraient aussi éviter de se montrer dogmatiques et devraient s'isoler de toute ingérence politique dans leur interprétation et leur présentation des résultats scientifiques.

9) Les médias d'information (et la presse populaire plus généralement) devraient s'efforcer de présenter les travaux scientifiques de manière aussi juste et instructive que possible, notamment en s'abstenant d'exagérer l'importance des résultats, de suggérer l'imminence de leur application ou d'extrapoler indûment les résultats au-delà de la portée de l'étude. Les journalistes devraient également situer les recherches dans le contexte de l'ensemble des données probantes disponibles et reconnaître, entre autres, les limites des méthodes particulières utilisées et la pertinence scientifique limitée des anecdotes, des témoignages et d'une étude en particulier.

10) Les chercheurs et les communicateurs scientifiques devraient toujours être conscients que les recherches sont susceptibles d'être interprétées d'une façon qui pourrait avoir, pour des personnes, des groupes ou des populations, des conséquences néfastes telles que des actes d'humiliation, de stigmatisation ou de racisme. Les communications devraient être faites en partenariat avec les participants aux recherches et leurs points de vue devraient être pris en compte tout au long du processus de recherche.

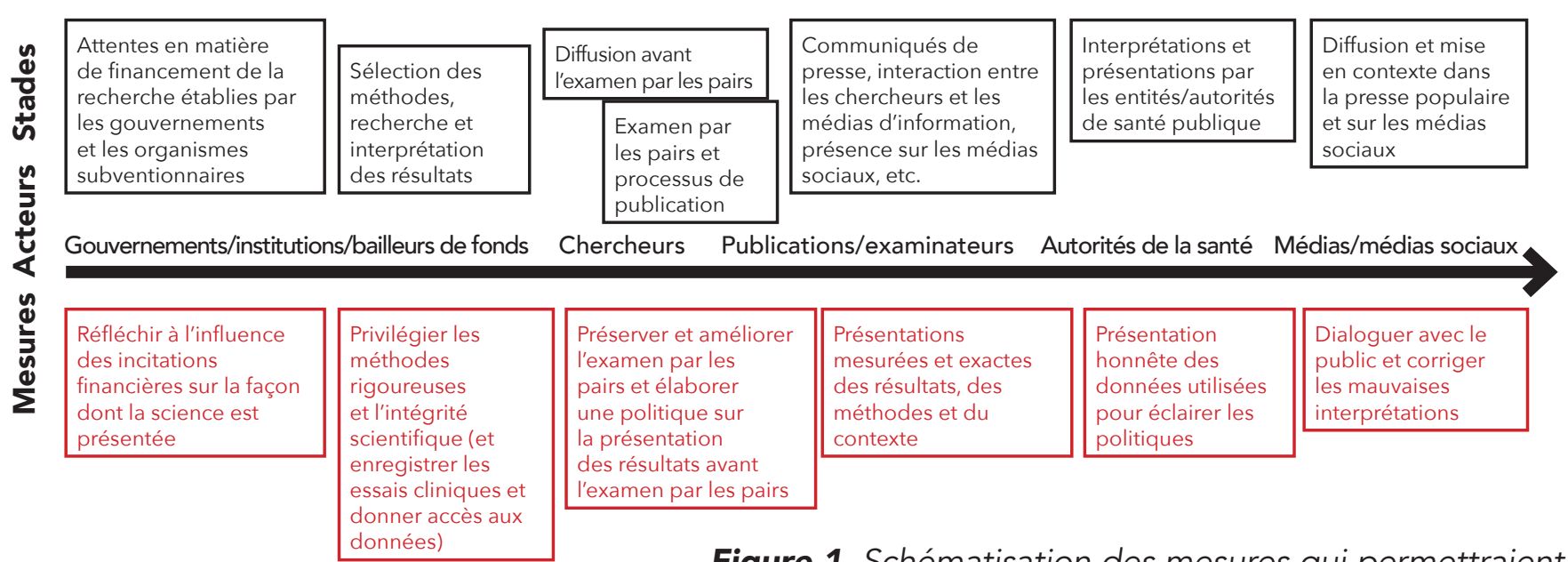

Figure 1. Schématisation des mesures qui permettraient d'améliorer la présentation des résultats scientifiques 
Abbas N, and Lamb S. 2020. A little science is a dangerous thing. Healthy Debate. 3 July. Available from https://healthydebate. ca/opinions/little-science-is-dangerous.

Abritis A, Marcus A, and Oransky I. 2020. An "alarming" and "exceptionally high" rate of COVID-19 retractions? Accountability in Research. Taylor and Francis Inc. DOI: 10.1080/08989621.2020.1793675.

Aksoy CG, Eichengreen B, and Saka O. 2020a. Revenge of the experts: Will COVID-19 renew or diminish public trust in science? European Bank for Reconstruction and Development. Working Paper No. 243 [online]: Available from https://www.ebrd.com/ publications/working-papers/revenge-of-the-experts.

Aksoy CG, Eichengreen B, and Saka O. 2020b. The Political Scar of Epidemics. Cambridge, MA. DOI: 10.3386/w27401.

Angus Reid Institute. 2020. COVID-19: Three-in-five worry about side-effects of a vaccine; many plan to take a "wait and see" approach. 4 August [online]: Available from http://angusreid.org/coronavirus-vaccine/.

Ball, P. 2015. "Novel, amazing, innovative": positive words on the rise in science papers. Nature News. DOI: 10.1038/ nature.2015.19024.

Balog-Way DHP and McComas KA. 2020. COVID-19: Reflections on trust, tradeoffs, and preparedness. Journal of Risk Research, O(0): 1-11. DOI: 10.1080/13669877.2020.1758192.

Baran NM, Goldman G, and Zelikova J. 2019. Abortion bans based on so-called "science" are fraudulent. Scientific American. 21 August [online]: Available from https://blogs.scientificamerican.com/observations/abortion-bans-based-on-so-called-scienceare-fraudulent/.

Bargain $\bigcirc$ and Aminjonov U. 2020. Trust and compliance to public health policies in time of COVID-19. Bordeaux Economics Working Papers [online]: Available from http://gretha.u-bordeaux.fr/LAREFlhttp://larefi.u-bordeaux.fr/.

Barry C, Han H, and McGinty B. 2020. Trust in science and COVID-19. Johns Hopkins Bloomberg School of Public Health Expert Insights. June [online]: Available from https://www.jhsph.edu/covid-19/articles/trust-in-science-and-covid-19.html.

Basch $\mathrm{CH}$, et al. 2020. News coverage of the COVID-19 pandemic: missed opportunities to promote health sustaining behaviors. Infection, Disease and Health. Australasian College for Infection Prevention and Control, 25(3): 205-209. DOI: 10.1016/j.idh.2020.05.001.

Bauchner H, Fontanarosa PB, and Golub RM. 2020. Editorial evaluation and peer review during a pandemic: how journals maintain standards. Journal of the American Medical Association, 324(5):453-454. DOI: 10.1001/jama.2020.11764.

Bazdaric K and Smart P. 2020. ESE and EASE call for high standards of research and editing. European Science Editing, 46: e53230. DOI: 10.3897/ese.2020.e53230.

Bell K and Green J. 2020. Premature evaluation? some cautionary thoughts on global pandemics and scholarly publishing. Critical Public Health, 30(4): 379-383. DOI: 10.1080/09581596.2020.1769406.

Blaming "overflow of manuscripts" and "obviously biased" reviewers, journal will retract homeopathy-COVID-19 paper. 2020. Retraction Watch [online]: Available from https://retractionwatch.com/2020/07/27/blaming-overflow-of-manuscripts-andobviously-biased-reviewers-journal-will-retract-homeopathy-covid-19-paper.

Boulware DR, et al. 2020. A randomized trial of hydroxychloroquine as postexposure prophylaxis for Covid-19. New England Journal of Medicine, 383(6): 517-525. DOI: 10.1056/NEJMoa2016638.

Bridgman A, et al. 2020. The causes and consequences of COVID-19 misperceptions: understanding the role of news and social media. Harvard Kennedy School Misinformation Review. Shorenstein Center for Media, Politics, and Public Policy, 1(3). DOI: 10.37016/mr-2020-028.

Bubela T. 2006. Science communication in transition: genomics hype, public engagement, education and commercialization pressures. Clinical Genetics, 70(5): 445-450. DOI: 10.1111/j.1399-0004.2006.00693.x.

Bubela T, Nisbet M, Borchelt R, et al. 2009. Science communication reconsidered. Nature Biotechnology, 27: 514-518. DOI: 10.1038/nbt0609-514.

Government of Canada. 2019. Policy on scientific and indigenous knowledge integrity [online]: Available from https://www. rcaanc-cirnac.gc.ca/eng/1575567784632/1575567805298.

Carleton researchers find Canadians most trust public health officials on COVID-19. 2020. Carleton Newsroom [online]: Available from https://newsroom.carleton.ca/2020/carleton-researchers-find-canadians-most-trust-public-health-officials-on-covid-19/.

Caulfield T, Sipp D, Murry CE, Daley GQ, Kimmelman J. 2016. Confronting stem cell hype. Science, 352(6287): 776-777. DOI: 10.1126/science.aaf4620.

Caulfield T. 2018. Spinning the genome: why science hype matters. Perspectives in Biology and Medicine, 61(4): 560-571. DOI: 10.1353/pbm.2018.0065.

Caulfield T. 2020. The COVID-19 pandemic will cause trust in science to be irreparably harmed. The Globe and Mail, 10 July [online]: Available from https://www.theglobeandmail.com/opinion/article-the-covid-19-pandemic-will-cause-trust-in-science-tobe-irreparably/. 
Caulfield T. Does Debunking Work? Correcting COVID-19 Misinformation on Social Media. [Preprint ]. DOI: 10.1186/s12916020-01556-3.

Caulfield T, and Condit C. 2012. Science and the sources of hype. Public Health Genomics, 15: 209-217. DOI: 10.1159/000336533.

Caulfield T, and Ogbogu U. 2015. The commercialization of university-based research: balancing risks and benefits. BMC Medical Ethics, 16(1): 70. DOI: 10.1186/s12910-015-0064-2.

Coronavirus: resources for reporters. 2020. First Draft [online]: Available from https://firstdraftnews.org/long-form-article/ coronavirus-resources-for-reporters/.

Coronavirus research publishing: the rise and rise of COVID-19 clinical trials. 2020. Natureindex.com [online]: Available from https://www.natureindex.com/news-blog/the-top-coronavirus-research-articles-by-metrics.

Council of Canadian Academies. 2010. Honesty, accountability and trust: fostering research integrity in Canada. DOI: ISBN 9781-926558-26-4.

Crow D, and Stacey K. 2020. Why is the "anti-vaxxer" movement growing during coronavirus pandemic? Los Angeles Times, 20 August [online]: Available from https://www.latimes.com/world-nation/story/2020-08-20/why-anti-vaxxer-movement-growingduring-coronavirus-pandemic.

Day M. 2020. Covid-19: experts criticise claim that remdesivir cuts death rates. BMJ: Clinical Research, 370: m2839. DOI: 10.1136/bmj.m2839.

Dearment A. 2020. Why hydroxychloroquine's appeal endures despite evidence it doesn't work for Covid-19. MedCity News [online]: Available from https://medcitynews.com/2020/08/why-hydroxychloroquines-appeal-endures-despite-evidence-itdoesnt-work-for-covid-19/?rf=1.

Devine D, Gaskell J, Jennings W, and Stoker G. 2020. Trust and the coronavirus pandemic: what are the consequences of and for trust? an early review of the literature. Political Studies Review, [preprint]: 1-12. DOI: 10.1177/1478929920948684.

Dinis-Oliveira RJ. 2020. COVID-19 research: pandemic versus "paperdemic", integrity, values and risks of the "speed science". Forensic Sciences Research, 5(2): 174-187. DOI: 10.1080/20961790.2020.1767754.

Downes SM, Leroy BP, Sharma SM, Sivaprasad S, and Dollfus H. 2020. Hydroxychloroquine hitting the headlines—retinal considerations. Eye,34: 1158-1160. DOI: 10.1038/s41433-020-0934-9.

Drage O'Reilly E. 2020. Retracted coronavirus studies are threatening trust in scientific data. Axios, June [online]: Available from https://www.axios.com/coronavirus-scientific-studies-trust-757f38eb-f2dd-40ab-8828-3b8fe43b5044.html.

Dupré J. 2020, "Following the science" in the COVID-19 pandemic. The Nuffield Council on Bioethics [online]: Available from https://www.nuffieldbioethics.org/blog/following-the-science-in-the-covid-19-pandemic.

Eisen MB, and Tibshirani R. 2020. How to identify flawed covid-19 research before it's too late. The New York Times, 20 July [online]: Available from https://www.nytimes.com/2020/07/20/opinion/coronavirus-preprints.html.

Everts S, and Greenberg J. 2020. New Carleton Study finds covid-19 conspiracies and misinformation spreading online. Carleton Newsroom [online]: Available from https://newsroom.carleton.ca/2020/new-carleton-study-finds-covid-19-conspiracies-andmisinformation-spreading-online/.

Finkbeiner A. 2018. The covert politics of cold-war science. Nature, 563: 32-33. DOI: 10.1038/d41586-018-07184-5.

Fleerackers A. 2020. Uncertainty in science communication: include it or lose it? Blog, Science Borealis [online]: Available from https://blog.scienceborealis.ca/uncertainty-in-science-communication-include-it-or-lose-it/.

Fraser N, Brierley L, Dey G, Polka JK, Pálfy M, and Coates JA. 2020. Preprinting a pandemic: the role of preprints in the COVID-19 pandemic. bioRxiv, [preprint]. DOI: 10.1101/2020.05.22.111294.

Fukuyama F. 2020. The pandemic and political order: it takes a state. Foreign Affairs [online]: Available from https://www. foreignaffairs.com/articles/world/2020-06-09/pandemic-and-political-order.

Gautret P, Lagier JC, Parola P, et al. 2020) 'Hydroxychloroquine and azithromycin as a treatment of COVID-19: results of an openlabel non-randomized clinical trial', International Journal of Antimicrobial Agents. Elsevier BV, 56(1):105949. DOI: 10.1016/j. ijantimicag.2020.105949. DOI:10.1016/j.ijantimicag.2020.105949.

Gerson J. 2020. Why that about-face on wearing masks is a problem. Maclean's, 8 April [online]: Available from https://www. macleans.ca/society/health/why-that-about-face-on-wearing-masks-is-a-problem/.

Di Girolamo N, and Meursinge Reynders R. 2020. Characteristics of scientific articles on COVID-19 published during the initial 3 months of the pandemic. Scientometrics, 125: 795-812. DOI: 10.1007/s11192-020-03632-0.

Glasziou PP, Sanders S, and Hoffmann T. 2020. Waste in covid-19 research. BMJ, 369:m1847. DOI: 10.1136/bmj.m1847. Gleick PH. 2020. Rushing science in the face of a pandemic is understandable but risky. Scientific American [online]: Available from https://www.scientificamerican.com/article/rushing-science-in-the-face-of-a-pandemic-is-understandable-but-risky/.

Goldberg MH Gustafson A, Maibach EW, Ballew MT, Bergquist P, Kotcher JE, Marlon JR, Rosenthal SA, and Leiserowitz A. 2020. Mask-wearing increased after a government recommendation: a natural experiment in the U.S. during the covid-19 pandemic. Frontiers in Communication, 5(44): 1-6 . DOI: 10.3389/fcomm.2020.00044. 
Gonsalves G. 2020. Statement from Yale faculty on hydroxychloroquine and its use in COVID-19. Medium, August [online]: Available from https://medium.com/@gregggonsalves/statement-from-yale-faculty-on-hydroxychloroquine-and-its-use-in-covid19-47d0dee7b2b0.

Gozzi N, Tizzani M, Starnini M, Ciulla F, Paolotti D, Panisson A, and Perra N. 2020. Collective response to the media coverage of COVID-19 pandemic on Reddit and Wikipedia. arXiv, [online preprint]: Available from http://arxiv.org/abs/2006.06446.

Green J, Edgerton J, Naftel D, Shoub K, and Cranmer SJ. 2020. Elusive consensus: polarization in elite communication on the COVID-19 pandemic. Science Advances, [preprint] 6(28): eabc2717. DOI: 10.1126/sciadv.abc2717.

Greenberg J, and Everts S. 2020. Pandemic puts public trust to the test. Policy Options Politiques, June [online]: Available from https://policyoptions.irpp.org/magazines/june-2020/pandemic-puts-public-trust-to-the-test/.

Greenhalgh T. 2020. Will COVID-19 be evidence-based medicine's nemesis? PLOS Medicine, 17(6): e1003266. DOI: 10.1371/ journal.pmed.1003266.

Hanage B, and Lipsitch M. 2020. How to report on the COVID-19 outbreak responsibly. Scientific American Blog Network, 23 February [online]: Available from https://blogs.scientificamerican.com/observations/how-to-report-on-the-covid-19-outbreakresponsibly/.

Herper M, and Riglin E. 2020. Data show panic, disorganization dominate the study of Covid-19 drugs. STAT, 6 July [online]: Available from https://www.statnews.com/2020/07/06/data-show-panic-and-disorganization-dominate-the-study-of-covid-19drugs/.

Hoffman J. 2020. "I won't be used as a guinea pig for white people." New York Times, 7 October [online]: Available from https://www.nytimes.com/2020/10/07/health/coronavirus-vaccine-trials-african-americans.html.

Jaklevic MC. 2020. Strong caveats are lacking as news stories trumpet preliminary COVID-19 research. healthnewsreview. org [online]: Available from https://www.healthnewsreview.org/2020/04/strong-caveats-are-lacking-as-news-stories-trumpetpreliminary-covid-19-research/.

Jensen JD, Carcioppolo N, King AJ, Bernat JK, Davis LS, Yale R, and Smith J. 2011 Including limitations in news coverage of cancer research: effects of news hedging on fatalism, medical skepticism, patient trust, and backlash. Journal of Health Communication, 16(5): 486-503. DOI: 10.1080/10810730.2010.546491.

Joseph A. 2020. Lancet, New England Journal retract Covid-19 studies, including one that raised safety concerns about malaria drugs. STAT [online]: Available from https://www.statnews.com/2020/06/04/lancet-retracts-major-covid-19-paper-that-raisedsafety-concerns-about-malaria-drugs/.

Journalists' resources for covering COVID-19. 2020. Science Media Centre of Canada [online]: Available from http:// sciencemediacentre.ca/site/journalists-resources-for-journalists-covering-covid-19/.

Kamenova K, and Caulfield T. 2015. Stem cell hype: media portrayal of therapy translation. Science Translational Medicine, 7(278): 1-4. DOI: 10.1126/scitransImed.3010496.

Kaplan JT, Gimbel SI, and Harris S. 2016. Neural correlates of maintaining one's political beliefs in the face of counterevidence. Scientific Reports, 6(1): 1-11. DOI: 10.1038/srep39589.

Kiley JP. 2020. NIH halts clinical trial of hydroxychloroquine. National Institutes of Health [online]: Available from https://www.nih. gov/news-events/news-releases/nih-halts-clinical-trial-hydroxychloroquine.

Kolopenuk J. 2020. Miskâsowin: Indigenous Science, Technology, and Society. Genealogy, 4(1): 21-38. DOI: 10.3390/ genealogy 4010021.

Kousha K, and Thelwall M. 2020. COVID-19 publications: database coverage, citations, readers, tweets, news, Facebook walls, Reddit posts. arXiv, [preprint]. DOI: 10.1162/qss_a_00066.

Kupferschmidt K. 2020. Three big studies dim hopes that hydroxychloroquine can treat or prevent COVID-19. ScienceMag.org, 9 June. DOI: 10.1126/science.abd2496.

Kupferschmidt K, and Cohen J. 2020. In plasma OK, critics see politics, not science. Science, 369(6507): 1038-1039. DOI: 10.1126/science.369.6507.1038.

LaFraniere S, Thomas K, Weiland N, Baker P, and Karni A. 2020. Scientists worry about political influence over coronavirus vaccine project. The New York Times, 2 August [online]: Available from https://www.nytimes.com/2020/08/02/us/politics/ coronavirus-vaccine.html.

Lane HC, and Fauci AS. 2020. Research in the context of a pandemic. New England Journal of Medicine, e2024638. DOI: 10.1056/NEJMe2024638.

Laurent L. 2020. Coronavirus: hydroxychloroquine farce has tragic consequences. Bloomberg Opinion, 8 June [online]: Available from https://www.bloomberg.com/opinion/articles/2020-06-08/coronavirus-hydroxychloroquine-farce-has-tragic-consequences. Leask J. 2020. Leaders can still build our trust to fight the virus - here's how. The Sydney Morning Herald, 26 March [online]: Available from https://www.smh.com.au/national/leaders-can-still-build-our-trust-to-fight-the-virus-here-s-how-20200324-p54die. html.

Ledford H. 2020. Chloroquine hype is derailing the search for coronavirus treatments. Nature, 580(7805): 573-573. DOI: 10.1038/d41586-020-01165-3. 
Leeming J. 2018. Why scientists should communicate hope whilst avoiding hype. Naturejobs Blog [online]: Available from http:// blogs.nature.com/naturejobs/2018/07/06/why-scientists-should-communicate-hope-whilst-avoiding-hype/.

Lep Ž, Babnik K, and Hacin Beyazoglu K. 2020. Emotional responses and self-protective behavior within days of the COVID-19 outbreak: the promoting role of information credibility. Frontiers in Psychology, 11(1846): 1-16. DOI: 10.3389/fpsyg.2020.01846. Limaye RJ, Sauer M, Ali J, Bernstein J, Wahl B, Barnhill A, et al. 2020. Building trust while influencing online COVID-19 content in the social media world. The Lancet Digital Health, e277-e278. DOI: 10.1016/S2589-7500(20)30084-4.

Liu M, Caputi TL, Dredze M, et al. 2020. Internet searches for unproven COVID-19 therapies in the United States. JAMA Internal Medicine, 180(8): 1116-1118. DOI: 10.1001/jamainternmed.2020.1764.

Liu Q, Zheng Z, Zheng J, Chen Q, Liu G, Chen S, Chu B, et al. 2020. Health communication through news media during the early stage of the covid-19 outbreak in China: digital topic modeling approach. Journal of Medical Internet Research, 22(4): e19118. DOI: $10.2196 / 19118$.

Locher C, Moher D, Cristea I, Florian N. 2020. Publication by association: the Covid-19 pandemic reveals relationships between authors and editors. MetaArXiv [preprint]. DOI: 10.31222/osf.io/64u3s.

London AJ, and Kimmelman J. 2020. Against pandemic research exceptionalism. Science, 368(6490): 476-477. DOI: 10.1126/ science.abc1731.

Lovari A. 2020. Spreading (dis)trust: covid-19 misinformation and government intervention in Italy. Media and Communication, 8(2): 458-461. DOI: 10.17645/mac.v8i2.3219.

Lovelace Jr B. 2020. Coronavirus: hydroxychloroquine prescription fills surged in March after Trump touted drug. CNBC News, 29 May [online]: Available from https://www.cnbc.com/2020/05/29/coronavirus-hydroxychloroquine-prescription-fills-surged-inmarch-after-trump-touted-drug.html.

Lowe D. 2019. The latest on drug failure and approval rates. ScienceMag.org [online]: Available from https://blogs.sciencemag. org/pipeline/archives/2019/05/09/the-latest-on-drug-failure-and-approval-rates.

Luth W, Jardine C, and Bubela T. 2013. When pictures waste a thousand words: analysis of the 2009 H1N1 pandemic on television news. PLoS ONE, 8(5): e64070. DOI: 10.1371/journal.pone.0064070.

Lwin MO, Lu J, Sheldenkar A, Schulz PJ, Shin W, Gupta R, Yang Y. 2020. Global sentiments surrounding the COVID-19 pandemic on Twitter: analysis of Twitter trends. JMIR Public Health and Surveillance, 6(2): p. e19447. DOI: 10.2196/19447.

Mahase E. 2020a. Covid-19: six million doses of hydroxychloroquine donated to US despite lack of evidence. BMJ, 368 : m1166. DOI: $10.1136 / \mathrm{bmj} . \mathrm{m} 1166$.

Mahase E. 2020b. Hydroxychloroquine for covid-19: The end of the line? BMJ, 369: m2378. DOI: 10.1136/bmj.m2378.

Majumder MS, and Mandl KD. 2020. Early in the epidemic: impact of preprints on global discourse about COVID-19 transmissibility. The Lancet Global Health, 8: e627-e630. DOI: 10.1016/S2214-109X(20)30113-3.

Mandavilli A. 2020. Scientists take aim at another coronavirus study in a major journal. The New York Times, 18 June [online]: Available from https://www.nytimes.com/2020/06/18/health/coronavirus-retractions-studies.html.

Massey DS, Opare MA, Wallach JD, Ross JS, and Krumholz HM. 2020. Assessment of preprint policies of top-ranked clinical journals. JAMA Network Open, 3(7): e2011127. DOI: 10.1001/jamanetworkopen.2020.11127.

Master Z, and Resnik DB. 2013. Hype and public trust in science. Science and Engineering Ethics, 19(2): 321-335. DOI: 10.1007/ s11948-011-9327-6.

Matthew D. 2020. French trust in science drops as coronavirus backlash begins. Times Higher Education, June [online]: Available from https://www.timeshighereducation.com/news/french-trust-science-drops-coronavirus-backlash-begins.

Matthews D. 2016. Academics "regularly lie to get research grants". Times Higher Education [online]: Available from https:// www.timeshighereducation.com/news/academics-regularly-lie-to-get-research-grants.

McGinley L, Abutaleb Y, Dawsey J, and Johnson CY. 2020. Inside Trump's pressure campaign on federal scientists over a covid-19 treatment. The Washington Post, 30 August [online]: Available from https://www.washingtonpost.com/health/ convalescent-plasma-treatment-covid19-fda/2020/08/29/e39a75ec-e935-11ea-bc79-834454439a44_story.html.

Medical Xpress. 2020. Lancet boosts review process after COVID study retraction. 18 September [online]: Available from https:// medicalxpress.com/news/2020-09-lancet-boosts-covid-retraction.html.

Mello MM, Greene JA, and Sharfstein JM. 2020. Attacks on public health officials during COVID-19. Journal of the American Medical Association, 324(8):741-742. DOI: 10.1001/jama.2020.14423.

Mendel A, Bernatsky S, Thorne JC, et al. 2020. Hydroxychloroquine shortages during the COVID-19 pandemic. Annals of the Rheumatic Diseases, [preprint]. DOI: 10.1136/annrheumdis-2020-217835.

Merkley E, Bridgman A, Loewen P, Owen T, Ruths D, and Zhilin O. 2020. A rare moment of cross-partisan consensus: elite and public response to the COVID-19 pandemic in Canada. Canadian Journal of Political Science. 53(2): 311-318. DOI: 10.1017/ S0008423920000311.

Mikkelson D. 2020. Is a global conspiracy promoting remdesivir over hydroxychloroquine for treating COVID-19? Snopes.com [online]: Available from https://www.snopes.com/fact-check/remdesivir-gilead-conspiracy/. 
Mulcahey T. 2020. 10 tips for journalists covering COVID-19. International Journalists' Network [online]: Available from https:// ijnet.org/en/story/10-tips-journalists-covering-covid-19.

Ontario Science Centre. 2017. Public trust in science news is dangerously low, new Ontario Science Centre study reveals. Cision, 18 September [online]: Available from https://www.newswire.ca/news-releases/public-trust-in-science-news-is-dangerously-lownew-ontario-science-centre-study-reveals-645328533.html.

Padilla J, and Hípola B. 2020. Ideology and polarization in times of coronavirus. LSE: Euro Crisis in the Press, June [online]: Available from https://blogs.Ise.ac.uk/eurocrisispress/2020/06/26/polarization-coronavirus/.

Pak A, and Adegboye OA. 2020. Whom shall i trust? salience of public trust in time of COVID-19 pandemic. [preprint online]. DOI: 10.13140/RG.2.2.15935.94884.

Palamenghi L, Barello S, Boccia S, and Graffigna G. 2020. Mistrust in biomedical research and vaccine hesitancy: the forefront challenge in the battle against COVID-19 in Italy. European Journal of Epidemiology, 35(8): 785-788. DOI: 10.1007/s10654-02000675-8.

Palayew A, Norgaard O, Safreed-Harmon K, et al. 2020. Pandemic publishing poses a new COVID-19 challenge. Nature Human Behaviour, 4(7): 666-669. DOI: 10.1038/s41562-020-0911-0.

Pang W, and Elkhodiry M. 2020. Hasty science in the battle against COVID-19 runs the risk of eroding public trust. The Star, 4 May [online]: Available from https://www.thestar.com/opinion/contributors/2020/05/04/hasty-science-in-the-battle-against-covid19-runs-the-risk-of-eroding-public-trust.html.

Pickles K, Cvejic E, Nickel B, et al. 2020. COVID-19: beliefs in misinformation in the Australian community. medRxiv, [preprint online]. DOI: 10.1101/2020.08.04.20168583.

Plackett B. 2020. Five better ways to assess science. Nature Index [online]: Available from https://www.natureindex.com/newsblog/five-better-ways-to-assess-science-research-metrics.

Porter C. 2020. The top doctor who aced the coronavirus test. The New York Times, 5 June [online]: Available from https://www. nytimes.com/2020/06/05/world/canada/bonnie-henry-british-columbia-coronavirus.html.

Pundi K, Perino AC, Harrington RA, Krumholz HM, Turakhia MP. 2020. Characteristics and strength of evidence of covid-19 studies registered on clinicaltrials.gov. JAMA Internal Medicine. DOI: 10.1001/jamainternmed.2020.2904.

Qaseem A, Yost J, Etxeandia-Ikobaltzeta I, and Humphrey LL. 2020. Update alert 2: should clinicians use chloroquine or hydroxychloroquine alone or in combination with azithromycin for the prophylaxis or treatment of COVID-19? living practice points from the American College of Physicians. Annals of Internal Medicine, 173(5): W88-W89. DOI: 10.7326/L20-1007.

Rabin RC. 2020. The pandemic claims new victims: prestigious medical journals. The New York Times, 14 July [online]: Available from https://www.nytimes.com/2020/06/14/health/virus-journals.html.

Ratcliff CL, Jensen JD, Christy K, Crossley K, and Krakow M. 2018. News coverage of cancer research: does disclosure of scientific uncertainty enhance credibility? In Risk and health communication in an evolving media environment. Edited by HD O'Hair. Taylor \& Francis Group, Milton Park. pp. 156-175 [online]: Available from https://www.taylorfrancis.com/ books/e/9781315168821/chapters/10.4324\%2F9781315168821-8.

Resnick B. 2019. Hyped-up science erodes trust. here's how researchers can fight back. Vox, 11 June [online]: Available from https://www.vox.com/science-and-health/2019/6/11/18652225/hype-science-press-releases.

Retracted coronavirus (COVID-19) papers. Retraction Watch [online]: Available from https://retractionwatch.com/retractedcoronavirus-covid-19-papers/.

Robinson P. 2020. Covid-19 poses trust issues for science. Chemistry World, May [online]: Available from https://www. chemistryworld.com/opinion/covid-19-poses-trust-issues-for-science/4011797.article.

Rosendaal FR. 2020. Review of: "Hydroxychloroquine and azithromycin as a treatment of COVID-19: results of an open-label non-randomized clinical trial Gautret et al 2010, DOI:10.1016/j.ijantimicag.2020.105949". International Journal of Antimicrobial Agents, 56(1), 106063 [online]: Available from https://linkinghub.elsevier.com/retrieve/pii/S0924857920302338.

Rutter H, Wolpert M, and Greenhalgh T. 2020. Managing uncertainty in the covid-19 era. BMJ - Opinion [online]: Available from https://blogs.bmj.com/bmj/2020/07/22/managing-uncertainty-in-the-covid-19-era/?fbclid=IwAR2mluBFP6KeBMcm5f3Fc jN_ULBF4Wu4Rv0iQAalBU6EQVLK5GxmTNjW3zc.

Saitz R, and Schwitzer G. 2020. Communicating science in the time of a pandemic. Journal of the American Medical Association, 324(5): 443-444. DOI: 10.1001/jama.2020.12535.

Sattui SE, Liew JW, Graef ER, Coler-Reilly A, et al. 2020. Swinging the pendulum: lessons learned from public discourse concerning hydroxychloroquine and COVID-19. Expert Review of Clinical Immunology, 16(7): 659-666. DOI: 10.1080/1744666X.2020.1792778.

Van Schalkwyk MCl, Hird TR, Maani N, Petticrew M, and Gilmore AB. 2020. The perils of preprints. BMJ, 370: m3111. DOI: 10.1136/bmj.m3111.

Schwitzer G. 2020. Why make international news out of 9 vague patient reports on remdesivir? HealthNewsReview.org [online]: Available from https://www.healthnewsreview.org/2020/07/why-make-international-news-out-of-9-vague-patient-reports-onremdesivir/. 
Seale H, Heywood AE, Leask J, Sheel M, Thomas S, Durrheim DN, Bolsewicz K, Kaur R. COVID-19 is rapidly changing: examining public perceptions and behaviors in response to this evolving pandemic. PloS one, 15(6): e0235112. DOI: 10.1371/ journal.pone.0235112.

Semeniuk I. 2018. Survey highlights tensions in public attitudes toward science. The Globe and Mail, 16 September [online]: Available from https://www.theglobeandmail.com/canada/article-survey-highlights-tensions-in-public-attitudes-toward-science/. Sheridan K. 2020. CDC director attempts to clarify controversial Covid-19 testing guidelines. STAT, 27 August [online]: Available from https://www.statnews.com/2020/08/27/redfield-clarify-controversial-testing-guidelines/?utm_ source=STAT+Newsletters\&utm_campaign=30cda33031-Daily_Recap\&utm_medium=email\&utm_term=0_8cab1d796130cda33031-116322369.

Silverman E. 2020. Most Americans see politics driving Covid-19 vaccine approval process', STAT, 31 August [online]: Available from https://www.statnews.com/pharmalot/2020/08/31/most-americans-believe-the-covid-19-vaccine-approvalprocess-is-driven-by-politics-not-science/?utm_source=STAT+Newsletters\&utm_campaign=44f091f0a7-MR_COPY_01\&utm_ medium=email\&utm_term=0_8cab1d7961-44f091f0a7-116322369.

Skipper CP, Pastick KA, Engen NW, et al. 2020. Hydroxychloroquine in nonhospitalized adults with early COVID-19: a randomized trial. Annals of Internal Medicine, [preprint]: M20-4207. DOI: 10.7326/M20-4207.

Statistics Canada. 2020. Crowdsourcing participants' trust in governments, public health authorities, businesses and others during the COVID-19 pandemic, [online]: Available from https://www150.statcan.gc.ca/n1/daily-quotidien/200626/dq200626beng.htm.

Steinberg I. 2020. Coronavirus research done too fast is testing publishing safeguards, bad science is getting through. The Conversation, April [online]: Available from https://theconversation.com/coronavirus-research-done-too-fast-is-testingpublishing-safeguards-bad-science-is-getting-through-134653.

Strategy for Patient-Oriented Research - Patient Engagement Framework. 2019. CIHR, Government of Canada [online]: Available from https://cihr-irsc.gc.ca/e/48413.html.

Strazewski L. 2020. How science communication is failing during COVID-19. American Medical Association - Public Health [online]: Available from https://www.ama-assn.org/delivering-care/public-health/how-science-communication-failing-duringcovid-19.

The First Nations Principles of OCAP, [online]: Available from http://www.fnigc.ca/ocap.html.

Thompson, C. 2020. Trump's hydroxychloroquine hype: a risk to my treatment and to him. STAT, 20 May [online]: Available from https://www.statnews.com/2020/05/20/hydroxychloroquine-trump-hype-jeopardize-supply-may-harm-him/.

Tingley K. 2020. Coronavirus is forcing medical research to speed up. The New York Times, 21 April [online]: Available from https://www.nytimes.com/2020/04/21/magazine/coronavirus-scientific-journals-research.html.

Toronto Sun. 2020. 'Editorial: the great Canadian mask flip-flop. 6 April [online]: Available from https://torontosun.com/opinion/ editorials/editorial-the-great-canadian-mask-flip-flop.

Tri-Council Policy Statement: Ethical Conduct for Research Involving Humans - TCPS 2 (2018). Government of Canada [online]: Available from https://ethics.gc.ca/eng/policy-politique_tcps2-eptc2_2018.html.

Troisi CL. 2020. I'm a public health researcher, and I'm dismayed that the CDC's missteps are causing people to lose trust in a great institution. The Conversation, 31 August [online]: Available from https://theconversation.com/im-a-public-healthresearcher-and-im-dismayed-that-the-cdcs-missteps-are-causing-people-to-lose-trust-in-a-great-institution-145236.

Tworek H, Beacock I, and Ojo E. 2020. Democratic health communications during Covid-19: a RAPID response. Vancouver. UBC Centre for the Study of Democratic Institutions, September.

U.S. Food \& Drug Admin. 2020. FDA cautions against use of hydroxychloroquine or chloroquine for COVID-19 outside of the hospital setting or a clinical trial due to risk of heart rhythm problems. [online]: Available from https://www.fda.gov/drugs/drugsafety-and-availability/fda-cautions-against-use-hydroxychloroquine-or-chloroquine-covid-19-outside-hospital-setting-or.

Udow-Phillips M, and Lantz PM. 2020. Trust in public health is essential amid the COVID-19 pandemic. Journal of Hospital Medicine, 15: 431-433 [preprint]. DOI: 10.12788/jhm.3474.

University of Oxford - News. 2020. No clinical benefit from use of hydroxychloroquine in hospitalised patients with COVID-19. 5 June [online]: Available from https://www.ox.ac.uk/news/2020-06-05-no-clinical-benefit-use-hydroxychloroquine-hospitalisedpatients-covid-19.

Urback R. 2020. Dr. Tam's about-face on masks damages trust at a crucial time. The Globe and Mail, 7 April [online]: Available from https://www.theglobeandmail.com/opinion/article-dr-tams-about-face-on-masks-damages-trust-at-a-crucial-time/.

Vaduganathan M, van Meijgaard J, Mehra MR, Joseph J, O’Donnell CJ, and Warraich HJ. 2020. Prescription fill patterns for commonly used drugs during the COVID-19 pandemic in the United States. Journal of the American Medical Association, 323(24): 2524-2526. DOI: 10.1001/jama.2020.9184.

Vinkers CH, Tijdink JK, and Otte WM. 2015. Use of positive and negative words in scientific PubMed abstracts between 1974 and 2014: retrospective analysis. BMJ, 351: 1-6. DOI: 10.1136/bmj.h6467.

Voss A. 2020. Statement on IJAA paper. International Society of Antimicrobial Chemotherapy [online]: Available from https:// www.isac.world/news-and-publications/official-isac-statement. 
Vuong QH. 2020. Reform retractions to make them more transparent. Nature, 582(7811): 149. DOI: 10.1038/d41586-02001694-x.

Weber B. 2019. Canadians' trust in science falling, poll suggests. CBC News, 23 September [online]: Available from https://www. cbc.ca/news/technology/science-survey-1.5291291.

WHO discontinues hydroxychloroquine and lopinavir/ritonavir treatment arms for COVID-19. 2020. WHO Newsroom [online]: Available from https://www.who.int/news-room/detail/04-07-2020-who-discontinues-hydroxychloroquine-and-lopinavir-ritonavirtreatment-arms-for-covid-19.

Wilson R. 2020. Trump pressure on health agencies risks undermining public trust. The Hill, 28 August [online]: Available from https://thehill.com/policy/healthcare/514053-trump-pressure-on-health-agencies-risks-undermining-public-trust.

Woolston C. 2014. Study points to press releases as sources of hype. Nature, 516(7531): 291. DOI: 10.1038/nature.2014.16551. Wysong, P. 2020. The need for rigor: retractions can damage public trust. Healthy Debate, July [online]: Available from https:// healthydebate.ca/2020/07/topic/retractions-damage-trust-covid19.

Yarborough M. 2014. Openness in science is key to keeping public trust. Nature, 515: 313. DOI: 10.1038/515313a.

Yavchitz A, Boutron I, Bafeta A, Marroun I, Charles P, Mantz J, and Ravaud P. 2012. Misrepresentation of randomized controlled trials in press releases and news coverage: a cohort study', PLoS Medicine, 9(9): e1001308. DOI: 10.1371/journal. pmed.1001308.

Yeo-Teh NSL, and Tang BL. 2020. An alarming retraction rate for scientific publications on Coronavirus Disease 2019 (COVID-19). Accountability in Research, 1-7 [preprint]. DOI: 10.1080/08989621.2020.1782203.

Zhang L, Tao Y, Shen M, Fairley CK, and Guo Y. 2020. Can self-imposed prevention measures mitigate the COVID-19 epidemic? PLoS Med, 17(7): e1003240, [online]: Available from https://doi.org/10.1371/journal.pmed.1003240.

Zheng Y, Goh E, and Wen J. 2020. The effects of misleading media reports about COVID-19 on Chinese tourists' mental health: a perspective article. Anatolia, 31(2): 337-340. DOI: 10.1080/13032917.2020.1747208. 